\title{
An Audio-Vocal Interface in Echolocating Horseshoe Bats
}

\author{
Walter Metzner \\ Department of Zoology, University of Munich, 8000 Munich, Germany
}

The control of vocalization depends significantly on auditory feedback in any species of mammals. Echolocating horseshoe bats, however, provide an excellent model system to study audio-vocal (AV) interactions. These bats can precisely control the frequency of their echolocation calls by monitoring the characteristics of the returning echo; they compensate for flight-induced Doppler shifts in the echo frequency by lowering the frequency of the subsequent vocalization calls (Schnitzler, 1968; Schuller et al., 1974, 1975).

It was the aim of this study to investigate the neuronal mechanisms underlying this Doppler-shift compensation (DSC) behavior. For that purpose, the neuronal activity of single units was studied during spontaneous vocalizations of the bats and compared with responses to auditory stimuli such as playback vocalizations and artificially generated acoustic stimuli. The natural echolocation situation was simulated by triggering an acoustic stimulus to the bat's own vocalization and by varying the time delay of this artificial "echo" relative to the vocalization onset.

Single-unit activity was observed before, during, and/or after the bat's vocalization as well as in response to auditory stimuli. However, the activity patterns associated with vocalization differed from those triggered by auditory stimuli even when the auditory stimuli were acoustically identical to the bat's vocalization. These neurons were called AV neurons. Their distribution was restricted to an area in the paralemniscal tegmentum of the midbrain.

When the natural echolocation situation was simulated, the responses of AV neurons depended on the time delay between the onset of vocalization and the beginning of the simulated echo. This delay sensitivity disappeared completely when the act of vocalization was replaced by an auditory stimulus that mimicked acoustic self-stimulation during the emission of an echolocation call. The activity of paralemniscal neurons was correlated with all parameters of echolocation calls and echoes that are relevant in context with DSC. These results suggest a model for the regulation of vocalization frequencies by inhibitory auditory feedback.

IKey words: bat echolocation, Doppler-shift compensation behavior, auditory feedback, vocalization control, single-unit electrophysiology, paralemniscal tegmentum, Rhinolophus rouxi]

\footnotetext{
Received May 19, 1992; revised Nov. 4, 1992; accepted Nov. 10, 1992.

I thank Drs. G. Schuller, E. Covey, G. Pollak, G. Neuweiler, W. Heiligenberg, M. Vater, J. Enright, C. Wong, and the anonymous reviewers for most valuable help and comments. Mrs. H. Tscharntke and A. Rau provided superb technical assistance. This study was supported by the Deutsche Forschungsgemeinschaft SFB 204/TP 10 (Schuller).

Correspondence should be addressed to Walter Metzner, University of California at Riverside, Department of Biology, Riverside, CA 92521-0427.

Copyright (C) 1993 Society for Neuroscience $0270-6474 / 93 / 131899-17 \$ 05.00 / 0$
}

One of the central questions in neuroscience is how sensory information about the environment is transformed into motor commands that guide appropriate behavior. To study sensorymotor integration, echolocating bats, and horseshoe bats in particular, provide an excellent model system. These bats precisely control the frequency of their echolocation calls in response to auditory feedback concerning the frequency of the returning echoes (Schnitzler, 1968).

The echolocation calls of horseshoe bats are emitted in a stereotyped manner and can easily be characterized. Each call is 100-115 dB sound pressure level (SPL) and consists of a long constant-frequency (CF) component and two short frequencymodulated (FM) parts, one at the beginning and one at the end of the call (Schnitzler, 1968; Neuweiler et al., 1987). Rufous horseshoe bats, Rhinolophus rouxi, the species used in this study, usually hang on a perch, scanning their environment for passing prey, which they then pursue and catch in flight with their wings or interfemoral membranes (Neuweiler et al., 1987). The frequency of the CF component emitted while the bat is perched is called the resting frequency (Schnitzler, 1968). The resting frequencies vary among bats, but in an individual the resting frequency is closely regulated around $78 \mathrm{kHz}$ and kept within $\pm 50 \mathrm{~Hz}$. The FM components cover a frequency range from the resting frequency to about $15 \mathrm{kHz}$ below (Fig. 1).

During flight, the frequency of the returning echo is shifted upward due to Doppler effects. This causes the CF component to return to the bat at a frequency higher than the emitted frequency. Since the auditory system of horseshoe bats is devoted to the representation of a very narrow frequency range that extends from the resting frequency to only about $300 \mathrm{~Hz}$ above it (Neuweiler, 1970), much of the information that is contained in the returning echo would be lost. Horseshoe bats detect the Doppler shift in the echoes and respond by lowering the frequency of their subsequent calls by an amount almost, but not exactly equal to the degree of Doppler shift. By adjusting the frequency of their emitted call, they maintain the CF component of the echoes within a very narrow range between 50 and $300 \mathrm{~Hz}$ above their normal resting frequency. This behavior is called Doppler-shift compensation (DSC), and the frequency at which they hold the echo frequency due to the compensation is called the reference frequency (Schnitzler, 1968). Like the resting frequencies, the reference frequencies also vary among bats, but for a given individual they are very stable and are maintained within a range of 100-200 Hz (Neumann and Schuller, 1991).

Horseshoe bats do not compensate for echoes unless they have a particular temporal and frequency relationships to the emitted call. Horseshoe bats will only compensate under the following conditions: (1) echoes must return within $20 \mathrm{msec}$ after the emission of an orientation call; (2) Doppler shifts must cause an increase in echo frequency, which occurs when the bat ap- 


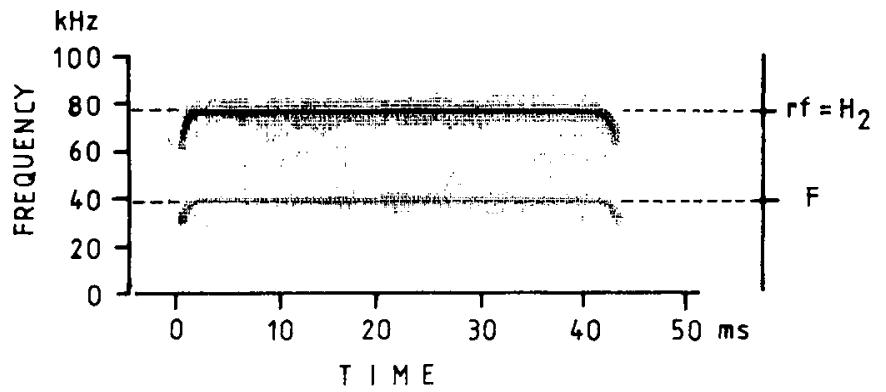

Figure 1. Representative echolocation call of the rufous horseshoe bat, Rhinolophus rouxi. The sounds consist of at least two harmonics and include a pure tone component with a duration of up to $50 \mathrm{msec}$. The pure tone component of the dominant second harmonic is around 78 $\mathrm{kHz}$. The fundamental or first harmonic $(F)$ is about $39 \mathrm{kHz}$, and is usually at least $20 \mathrm{~dB}$ less intense than the second harmonic $\left(\mathrm{H}_{2}\right)$. In nonflying bats, the emitted pure tone frequency of the main (second) harmonic is called the "resting frequency" $(r f)$. The frequency (ordinate) is plotted against the duration (abscissa) of the echolocation call; the relative intensity of the different frequency components is expressed by the darkness of shading. The data were analyzed using a computer program written by M. Obrist (Obrist, 1989).

proaches a background target; (3) Doppler shifts must not be greater than 4-5 kHz, which corresponds to the maximum Doppler shift that can be generated by the horseshoe bat's flight spced (Schnitzler, 1968; Schuller, 1974, 1977; Schuller et al., 1974, 1975; Schuller and Suga, 1976; Trappe and Schnitzler, 1986; Neumann and Schuller, 1991).

Within the bat's auditory system, neurons that are tuned within the narrow frequency range between the resting frequency and about $300 \mathrm{~Hz}$ above it show many specializations. They are characterized by their sharp tuning curves that have $Q_{10 \mathrm{~dB}}$ values as high as 400 . These neurons are numerically and spatially overrepresented at all levels of the auditory system from the periphery to the cortex [e.g., Bruns, 1976; Neuweiler and Vater, 1977 (cochlear nucleus); Schuller and Pollak, 1979 (inferior colliculus); Ostwald, 1984 (auditory cortex); Vater et al., 1985 (cochlea); Metzner and Radtke-Schuller, 1987 (nuclei of the lateral lemniscus); Casseday et al., 1988 (superior olivary complex)]. Thus, horseshoe bats adjust their voices to ensure that an echo of interest will stimulate a region of the cochlea innervated by a disproportionately large neuronal population that have exceptionally sharp tuning curves. This behavior has been compared with visual fixation, where eye movements keep an image of interest centered on the fovea, a region of the retina having densely packed receptors and neurons with small receptive fields (Schuller and Pollak, 1979). Because of this analogy with the visual system, the sharply tuned overrepresented neurons of the horseshoe bat's auditory nerve are referred to as the auditory fovea. The portions of the central auditory system that represent that region of the cochlea are referred to as the foveal representation, or simply also as the auditory fovea.

During DSC, the bat regulates the vocalized frequencies to within $0.1 \%$ of the CF component of the emitted calls. This represents one of the most precise forms of sensory-motor integration known. The purpose of the present study was to investigate the properties of the neurons that provide an interface between the processing of acoustic signals and the motor control of vocalization. Particular attention is given to neurons clustered in an area of the paralemniscal tegmentum of the mesencephalon. Neurons in this paralemniscal area were either inhibited or excited by the act of vocalization, and responded to acoustic stimuli that mimicked the emitted calls differently from the responses observed when the bat emitted calls. The discharge patterns and the discharge rates of paralemniscal neurons are correlated with almost all parameters of echolocation calls and echo signals that occur during DSC. Based on the unique features of these neurons, a model for the regulation of vocalization frequencies due to auditory feedback is proposed.

Short reports of parts of these results were presented previously (Metzner, 1988a,b, 1989).

\section{Materials and Methods}

A total of seven rufous horseshoe bats, Rhinolophus rouxi, from $\mathrm{Sri}$ Lanka (six females and one male) were used in this study. Their Dopplershift compensation bchavior was tested using a method described by Schuller et al. (1974). The experimental animals vocalized spontaneously and compensated fully for simulated Doppler shifts up to $4 \mathrm{kHz}$ above their resting frequencies. The resting frequencies of the bats ranged between $76.1 \mathrm{kHz}$ and $79.0 \mathrm{kHz}$ for the females and was $74.5 \mathrm{kHz}$ for the male. The intraindividual variation was within $\pm 100 \mathrm{~Hz}$ during the experimental period of 2-5 weeks per bat.

The bats were anesthetized with halothane for surgical preparation, and the surgical incisions were infiltrated with local anesthetic (Novocain). During the recording sessions the bats were fully awake. The preparation and recordings were made under stereotaxic methods described in detail by Schuller et al. (1986). Briefly, the head of the animal was fixed by a small metal tube attached to the dorsally exposed skull with glue and dental cement. The mounting system was designed to allow reproduciblc positioning of the head in a stereotaxic device for repeated recording sessions with the same animal. Measurements of the skull profile were made, which overlay the region of the inferior colliculus and $500 \mu \mathrm{m}$ either side of the midline. Since the position of the brain below the skull was known, it was possible to determine the coordinates of the target area in the brain. A stereotaxic atlas permitted location of the desired position for a hole on the dorsal skull in order that electrode penetrations would be in the target area.

Electrode penetrations entered through a single point above the caudal two-thirds of the inferior colliculus. The nuclei of the lateral lemniscus, and the adjacent tegmental areas were sampled systematically by changing the penetration angle in steps of $3-7^{\circ}$ in the mediolateral and rostrocaudal directions.

To verify the electrode positions, electrolytic lesions ( $1 \mu \mathrm{A}$ for $2 \mathrm{~min}$ ) were made at the end of the cxpcriment. Additionally, injections of wheat germ agglutinated to horseradish peroxidase (WGA-HRP) were placed in four bats primarily to trace projections. These injection sites, however, were also used for verification purposes. Comparison of the actual location of a lesion or injection site with the position predicted from the stereotaxic coordinates showed an accuracy of at least \pm 150 $\mu \mathrm{m}$.

Single-unit recordings were made in an anechoic chamber. During recording, bats vocalized spontaneously. Echoes reflected off surrounding objects were below the noise level, that is, below $40 \mathrm{~dB}$ SPL when measured at the position of the bat's head with a $1 / 4$ inch condenser microphone (Brïel and Kjaer type 4135), and subsequent preamplifier (BandK type 2618) and amplifier (BandK type 2610). It cannot be excluded that such weak echoes may have affected the measurement of minimum response thresholds and of intensity spike count functions at intensity levels below $40 \mathrm{~dB}$ SPL. However, since all other neuronal response properties, such as responses to playback vocalizations or to echo mimics, were examined at intensity levels far above $40 \mathrm{~dB}$ SPL (usually between 85 and $95 \mathrm{~dB}$ SPL; see below), the effect of these echoes became negligible. The temperature in the experimental recording chamber was maintained between $25^{\circ} \mathrm{C}$ and $30^{\circ} \mathrm{C}$. The acoustic stimuli were presented under free-field conditions. The condenser loudspeaker was positioned at an horizontal angle of $30^{\circ}$ lateral and $10^{\circ}$ ventral to the axis perpendicular to the horseshoe of the nose-leaf. This corresponds to the angle of maximal sensitivity of the horseshoe bat's ears (Pietsch and Schuller, 1987). Sound was usually delivered from the side contralateral to the recording side. The distance between the loudspeaker and the bat's closest ear was $15 \mathrm{~cm}$. The loudspeaker was calibrated using a 1/4 inch condenser microphone (Brüel and Kjaer type 4135) with subsequent amplifiers (preamplifier $B \& K$ type 2618 and amplifier B\&K type 2610). The microphone was positioned at a distance of 15 
$\mathrm{cm}$ from the loudspeaker. The loudspeaker had a flat frequency response from 10 to $100 \mathrm{kHz}( \pm 3 \mathrm{~dB})$.

The spontaneous vocalizations of the bats were recorded with a $1 / 4$ inch condenser microphone (distance from the bat's nostrils, ca. $15 \mathrm{~cm}$ ) and subsequent amplifiers. They were played back via a transient recorder with a variable output delay of up to $63 \mathrm{msec}$ (64 kilobyte memory, $1 \mathrm{MHz}$ sample rate). The natural echolocation situation was simulated by triggering an acoustic stimulus (either playback or artificial signals) to the onset of the orientation call and by varying the time interval between simulated echo and preceding echolocation sound. The frequency of the playback stimulus could be changed in order to produce artificial Doppler shifts (Schuller et al., 1974). Additional acoustic stimuli were generated artificially as described elsewhere (Metzner and RadtkeSchuller, 1987). These artificial stimuli included pure tone pulses and $\mathrm{CF}$ signals with frequency-modulated onsets and offsets similar to the bat's echolocation calls (Neuweiler et al., 1987). The frequencies tested varied between 10 and $110 \mathrm{kHz}$; the intensities ranged from $-10 \mathrm{~dB}$ SPL to $110 \mathrm{~dB}$ SPL. When vocalization was simulated acoustically, either by playback or by artificially generated acoustic stimuli, its harmonic composition was retained (Neuweiler et al., 1987). Both the playback of the bat's own calls and the artificial vocalization mimics were delivered at intensities of 85-95 dB SPL, which corresponds to intensities that reach the bat's ear when emitting pulses (Pietsch and Schuller, 1987).

For recording neuronal activity, glass micropipettes, pulled to tip diameters smaller than $1 \mu \mathrm{m}$ and filled with $3 \mathrm{M} \mathrm{KCl}$ (impedances, 3$5 \mathrm{M} \Omega$ ), were used. The recording electrodes were positioned on the brain surface under visual control, and advanced by a microdrive unit. Extracellular neuronal discharges were amplified and filtered by conventional techniques and fed on line to the laboratory computer (LSI 11/ $23 \mathrm{DEC}$ ) to generate dot-raster and/or poststimulus time histograms, covering various periods of vocalizations or acoustic-stimulus presentations. The bin width used for data collection was $1 \mathrm{msec}$. Only single units were recorded. Fiber recordings were distinguished from those of neurons by spike shape and amplitude. Respiration was monitored by an anemometer employing a thermistor element. No correlation was found between neuronal activity and the respiration cycle except when the bat's expiration was accompanied by the emission of an echolocation call. During recording, the duration and the frequency of the CF portion of the bat's vocalizations, and the beginning and the end of the expiration were monitored on line by the computer. All programs were written by M. Betz, University of Munich.

The reconstruction of all electrode penetrations revealed that the units recorded were distributed evenly and can be considered a random sample of the neurons present. Typically, during a recording session the neuronal activity associaled with the spontaneous vocalization of the bat was recorded, and then activity elicited by playback of vocalization (time delay fixed at $63 \mathrm{msec}$ ) was tested. Then, the unit's tuning curve and best frequency, spike count function, and discharge patterns during single- and double-pulse stimulations were determined. Off-line analysis yielded $Q_{\text {Ind }}$ values, latency at $30-40 \mathrm{~dB}$ above minimum threshold, effect of different duration and CF frequency of the vocalization, and the unit's response to varying the time delay between the onset of spontaneous or simulated vocalization and echo mimic.

\section{Results}

A total of 755 single units were recorded in the midbrain and brainstem of seven rufous horseshoe bats, Rhinolophus rouxi. Units were located in the inferior colliculus (Fig. 2, IC; 223 units), the nuclei of the lateral lemniscus (NLL; 95 units), and adjacent tegmental structures (437 units). Of these tegmental units, $\mathbf{3 3 5}$ were clustered in an area in the midbrain tegmentum just rostral and medial to the nuclei of the lateral lemniscus (Fig. 2). This "paralemniscal tegmentum" is enclosed within the lemniscal fiber bundle and extends from approximately $500 \mu \mathrm{m}$ rostral to the nuclei of the lateral lemniscus over a distance of about $1400 \mu \mathrm{m}$ caudally toward a position medial to the dorsal nucleus of the lateral lemniscus (DNLL) (Fig. 3). These paralemniscal neurons are the units described in this study.

The "paralemniscal tegmentum," as delineated here, is defined only physiologically by the occurrence of neurons de- scribed in detail below. Whether this paralemniscal area in the midbrain tegmentum is homolog to the paralemniscal zone as described elsewhere (e.g., cat, Henkel and Edwards, 1978; rat, Paxinos and Watson, 1986; mustached bat, Covey et al., 1987) is not a subject of this article.

Paralemniscal neurons can be divided into three major groups based on their responses to emitted echolocation calls and acoustic stimuli that mimic the echolocation calls. The first type, called auditory neurons, responded to tone bursts with discharge rates and patterns that were highly similar to the responses observed when the bat emitted echolocation calls; 118 units (35\%) of this type were observed in the paralemniscal tegmentum. The second type, nonauditory units, were spontaneously active but they could not be driven by any acoustic stimulus or by emitted calls; 28 units ( $8 \%$ ) of this type were recorded within the paralemniscal tegmentum. The third type, called audiovocal (AV) neurons, are the main focus of this report. AV neurons were either inhibited or excited by the act of vocalization, and responded to acoustic stimuli that mimicked the emitted calls in a manner that was both qualitatively and quantitatively different from the responses observed when the bat emitted calls. Their differential response to an emitted call compared to an identical signal played back to the bat was the distinguishing feature of $\Lambda \mathrm{V}$ neurons; $189 \mathrm{\Lambda V}$ units were recorded and made up $56 \%$ of the paralemniscal neurons that were recorded. The location of all AV neurons was confined to the paralemniscal tegmentum (Fig. 2).

\section{AV units}

All AV units were spontaneously active, and the spontaneous activity was cither suppressed or cnhanced by vocalizations. In addition, AV neurons responded in a variety of different ways to acoustic stimuli presented from a loudspeaker. Based on these features, AV units were subdivided into five distinct types.

(1) VOC inhibition units. VOC inhibition units were characterized by a suppression of spontaneous activity prior to and during vocalization. These were the most common type of AV unit and constituted $50 \%$ of the AV neurons recorded (94 out of 189). It is of particular significance that the suppression of background activity was not correlated in time with any auditory stimulus, but rather was correlated in time with the act of vocalization. When acoustic signals that mimicked the echolocation calls were presented, the majority of these units were excited and responded with a tonic discharge pattern.

These features are illustrated by the unit in Figure 4. Prior to pulse emission this unit was spontaneously active (Fig. $4 A$ ), but during vocalization the background activity was suppressed. The suppression began up to $5 \mathrm{msec}$ before the onset of vocalization, and ended with the termination of the vocalization. Figure $4 A$ also shows that when the echolocation call was played back to the bat, just after pulse emission, the unit responded with a tonic firing pattern. The discharges evoked by the playback signal had latencies of $5.1 \pm 1.9 \mathrm{msec}$ on average. Figure $4 B$ shows that when the playback vocalization was presented so that it overlapped in time with the emitted orientation call, the suppression due to the bat's vocalization was interrupted by discharges evoked by the playback signal.

The excitation evoked by the playback signal was independent of and overrode any effect due to the act of vocalization. This is illustrated in Figure $4 C$, which shows the discharge evoked by an acoustic signal delivered when the bat was not emitting echolocation calls. The stimulus in this case was an electroni- 
Figure 2. Schematic representation of the spatial distribution of neurons recorded within the deep layers of inferior colliculus $(I C)$, the nuclei of the lateral lemniscus $(N L L)$, and the adjacent tegmentum. $A-F$ represent frontal sections through the brain at the levels indicated in the parasagittal reconstruction in the inset at top right. The paralemniscal tegmentum $(P L)$ is marked by the dark area. Not all neurons recorded from the inferior colliculus are shown here. AV units (open circles), auditory neurons (solid circles), and neurons showing activity correlated neither to the bat's vocalization, to expiration, nor to auditory stimuli (crosses). $S C$, superior colliculus; $P A G$, periaqueductal gray; $I C R$, rostral pole of the inferior colliculus; $I C C$, central nucleus of the inferior colliculus; $D N L L$, dorsal nucleus of the lateral lemniscus; $I N L L$, intermediate nucleus of the lateral lemniscus; $V N L L$, ventral nucleus of the lateral lemniscus; $C N$, cochlear nucleus; $I C X$, external nucleus of the inferior colliculus.

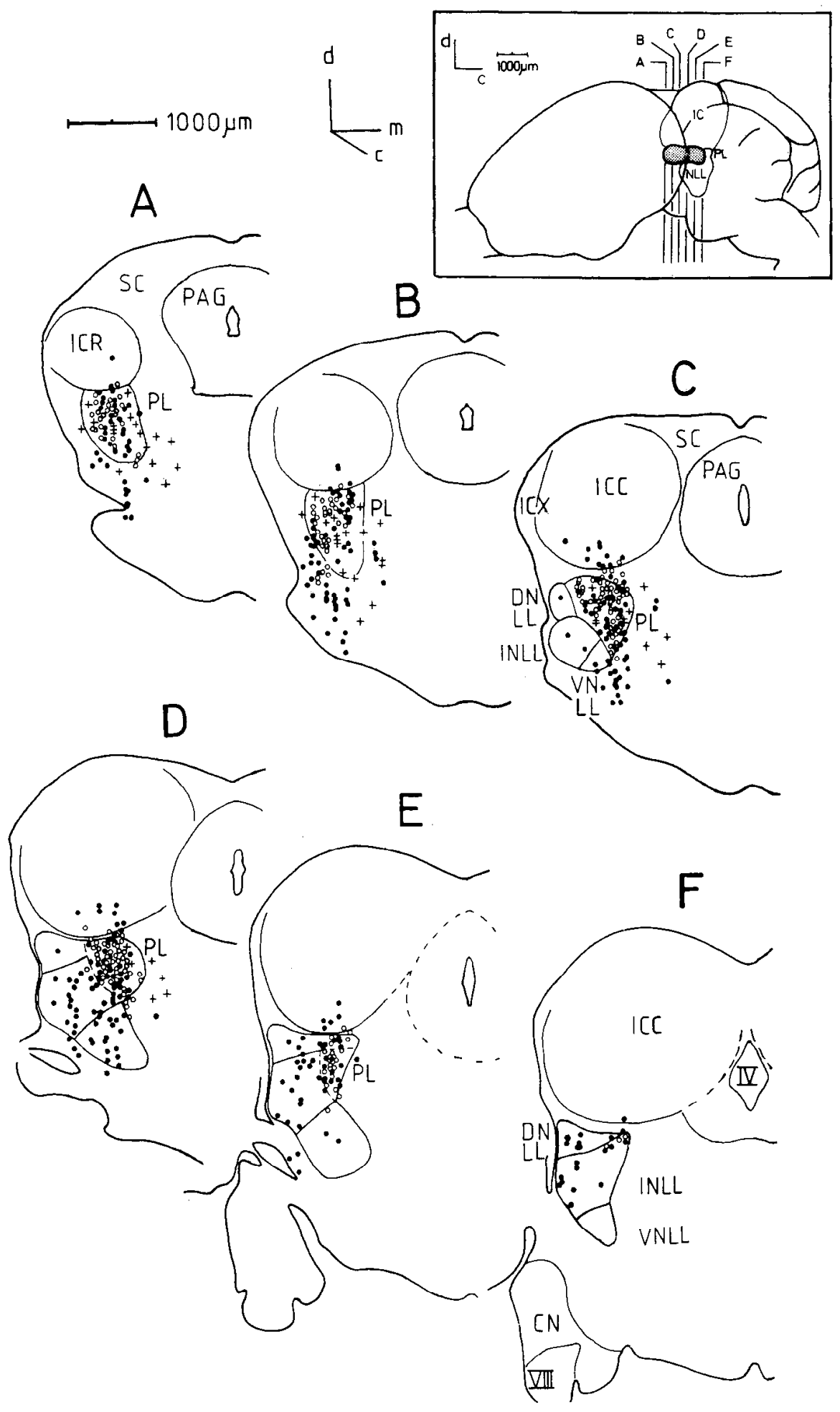

Figure 3. Histological view of the paralemniscal tegmentum in frontal sections. $A$, Nissl-stained frontal section through the midbrain of a horseshoe bat. The brain structures are schematically indicated in the right panel. The rectangle indicates the enlarged portions shown in $B$ and $C$. $B$, Enlarged view of the nuclei of the lateral lemniscus and the paralemniscal tegmentum in Nissl-stained section. $C$, Fiber-stained section through approximately the same part of the midbrain as shown in $B$. The section is situated about $50 \mu \mathrm{m}$ adjacent to the section shown in $A$ and $B$. $c n$, cochlear nucleus; $d n l l$, dorsal nucleus of the lateral lemniscus; icc, central nucleus of the inferior colliculus; icx, external nucleus of the inferior colliculus; inll, intermediate nucleus of the lateral lemniscus; $v n l l$, ventral nucleus of the lateral lemniscus; ncat, nucleus of the central acoustic tract; pag, periaqueductal gray; $p l$, paralemniscal tegmentum. Scale bars: $A, 1000 \mu \mathrm{m} ; B$ and $C, 100 \mu \mathrm{m}$. 

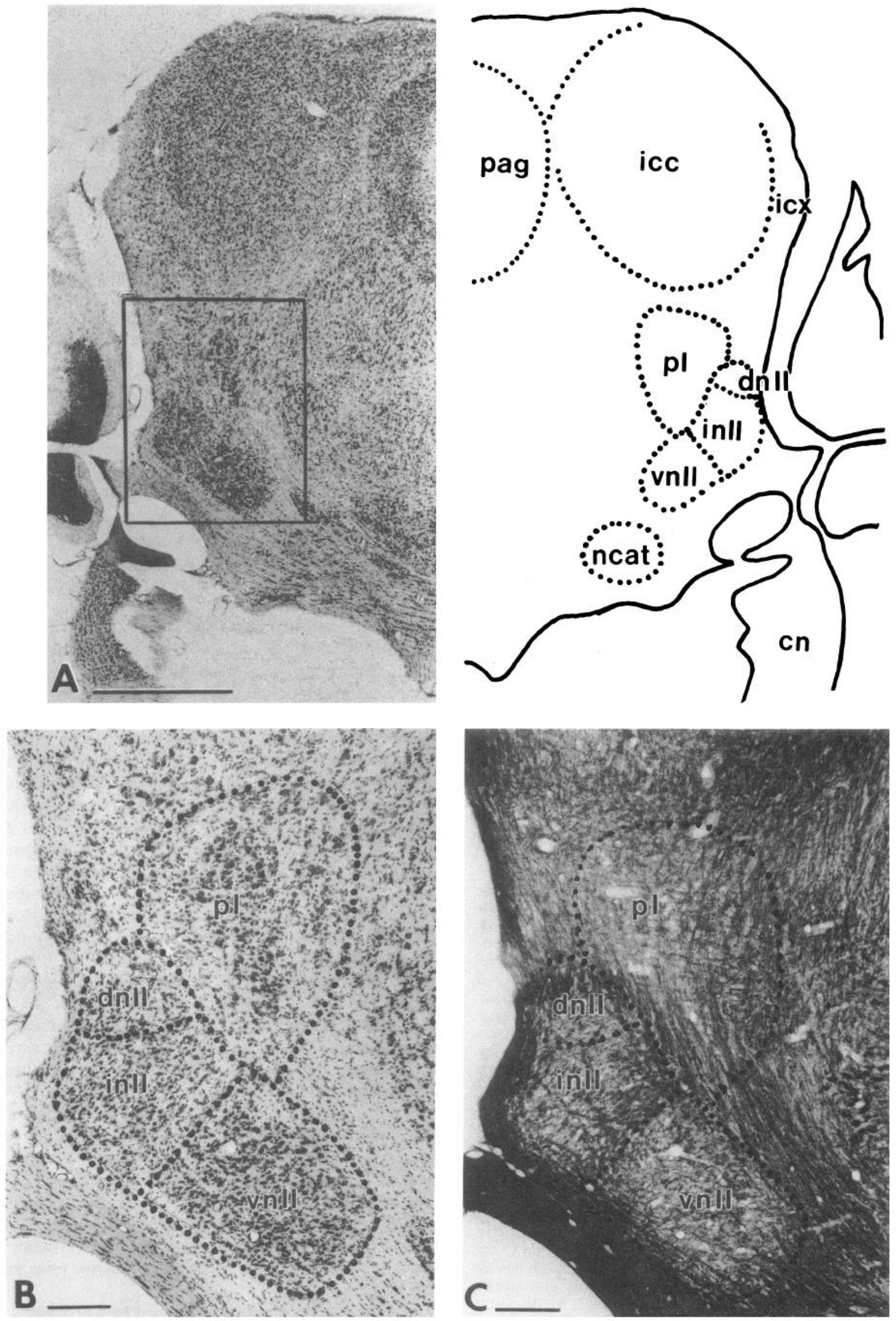

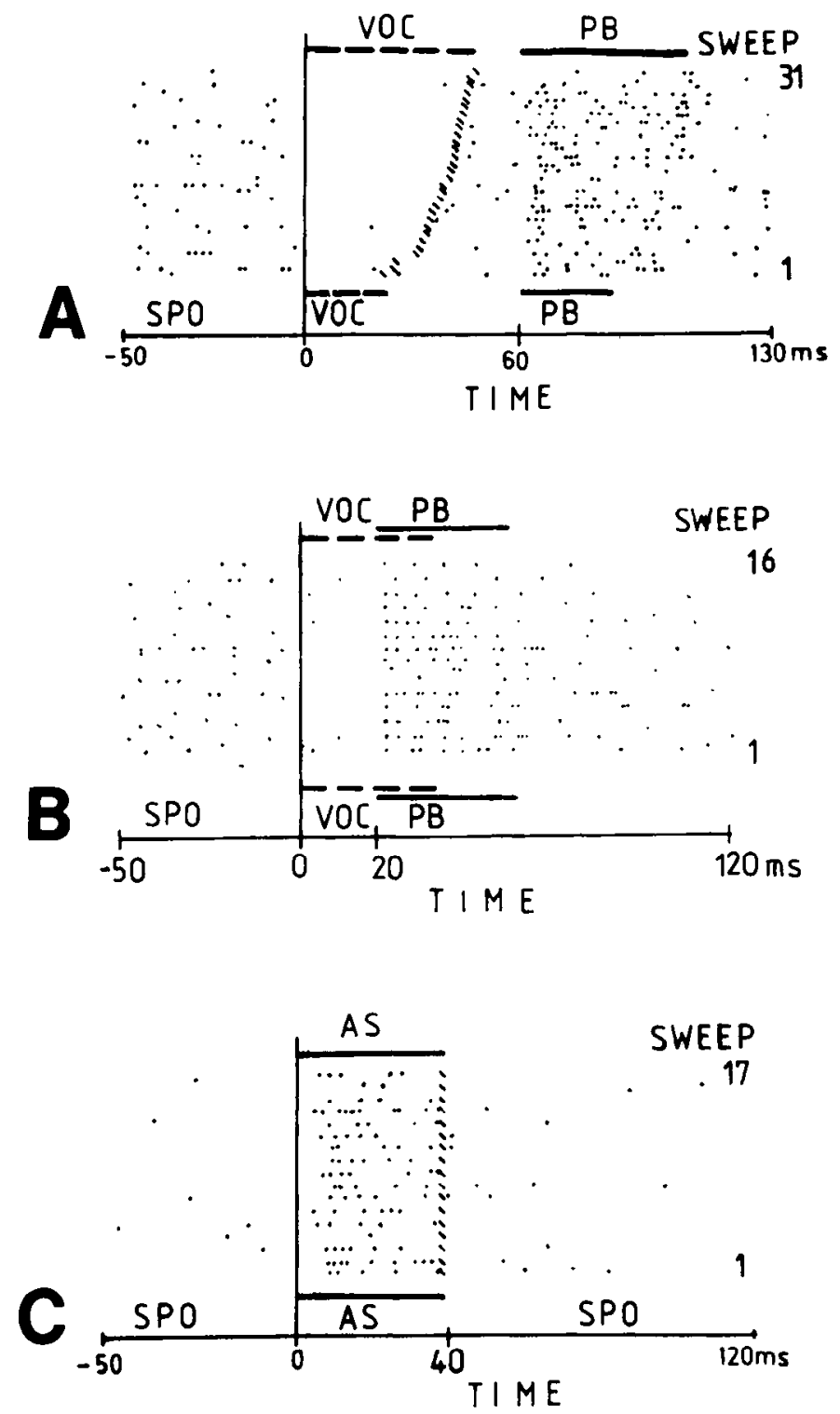

Figure 4. "VOC inhibition" neuron: raster displays of spike occurrences (dots) as a function of time (abscissa) for different stimulus conditions. $A$, The neurons were spontaneously active. During the bat's vocalization $(V O C)$ their background activity $(S P O)$ was suppressed, while playback of vocalizations $(P B)$ elicited excitation. Vocalizations are arranged according to incrcasing vocalization durations from bottom (SWEEP I) to top (SWEEP 31). Vocalizations start at the vertical line (marked by " 0 msec" on the time axis), and end at slashes. The time delay of the playback stimuli was $60 \mathrm{msec}$, the playback frequency was identical to the bat's vocalization frequency, and the playback intensity was $20 \mathrm{~dB}$ less than the call intensity. The shortest and the longest vocalizations are indicated by an interrupted bar at the bottom and at the top, respectively. In the same way, the shortest and the longest playback stimulus is indicated by a continuous bar. $B$, When a playback stimulus temporally overlapped with the bat's vocalization, the auditory response to this playback dominated over the inhibition during sound emission. Playback delay, $20 \mathrm{msec}$; other stimulus conditions were as in $A . C$, Artificially generated auditory stimuli $(A S)$, which were acoustically identical to echolocation calls but were presented independently of the bat's vocalization elicitcd cxcitatory responses also. Consecutive stimuli (17 sweeps) are presented from the bottom (SWEEP 1) to the top (SWEEP 17). The stimuli were harmonically composed matching the harmonic composition of the bat's echolocation call (intensity of fundamental, $65 \mathrm{~dB}$ SPL; intensity of second harmonic, $85 \mathrm{~dB}$ SPL). The stimuli started at the vertical bar and ended at the slashes. Their duration was $40 \mathrm{msec}$ as indicated by the bars at the bottom and at the top. cally generated CF signal composed of both the fundamental (= first harmonic, $39 \mathrm{kHz})$ and second harmonic $(78 \mathrm{kHz})$ of the bat's echolocation call. This mimic of the bat's orientation call elicited the same discharge pattern as the playback of the original vocalization that was presented either after pulse emission or while the pulse was being emitted (compare Fig. $4 C$ with $4 A, B$ ).

However, the level of spontaneous activity was higher during periods of time when the bat was vocally active and emitting one or several series of echolocation calls than during those time periods when the bat was unwilling to vocalize [compare spontaneous activity (SPO) in Fig. $4 A, B$ with Fig. $4 C$ ]. Within a series of echolocation pulses, VOC inhibition neurons fired on average at a rate of 59 spikes/sec between subsequent vocalization calls, while when the bat was silent for more than $1 \mathrm{~min}$ the level of spontaneous activity dropped to 19 spikes/sec.

The VOC inhibition units had some response characteristics that could play a role in monitoring Doppler-shifted echoes. The best frequencies of 90 of the 94 VOC inhibition neurons were sharply tuned within a frequency range from $150 \mathrm{~Hz}$ above the bat's individual resting frequency to $5 \mathrm{kHz}$ above it (Fig. $5 A$ ). In fact, $55 \%$ of the VOC inhibition population had best frequencies clustered in a narrow band only $1350 \mathrm{~Hz}$ widc that varied from 150 to $1500 \mathrm{~Hz}$ above the individual bat's resting frequency. Four VOC inhibition units had best frequencies at or only up to $1 \mathrm{kHz}$ below the resting frequency. No VOC inhibition neuron had best frequencies between the resting frequency and $150 \mathrm{~Hz}$ above. Thus, the best frequencies of VOC inhibition neurons corresponded closely to the reference frequencies of the bats and to the range of echo frequencies that would elicit DSC.

VOC inhibition units were sharply tuned to frequency, although the average tuning curve was not as narrow as those of units tuned to comparable frequencies in the primary auditory pathway. Sharpness of tuning was evaluated by measuring the $Q_{10 \mathrm{~dB}}$ value, defined as the range of frequencies to which the unit responds at a level $10 \mathrm{~dB}$ above its minimum threshold divided by its best frequency; the higher the $Q_{10 \mathrm{~dB}}$ value, the sharper the tuning. The $Q_{1 \mathrm{~dB}}$ values of VOC inhibition neurons were 29.5 \pm 2.3 on average. For comparison, $Q_{10 \mathrm{~dB}}$ values of neurons of the nucleus of the lateral lemniscus in comparable frequency ranges were around 200 (Metzner and Radtke-Schuller, 1987). The tuning curves were generally asymmetric, with a shallow slope directed toward the higher frequencies and a much steeper slope for frequencies below the best frequency (Fig. $5 B$ ). The shapes of the tuning curves were similar to those reported for units tuned to the same range of frequencies in the horseshoe bat's primary auditory pathway, and both are unusual in that tuning curves for units tuned to other frequencies have sharp slopes on the high-frequency side and shallower slopes on the low-frequency side (Metzner and Radtke-Schuller, 1987).

The firing rate of VOC inhibition neurons changed dramatically with very small frequency shifts around the unit's best frequency. This feature is shown in Figures $5 C$ and 6 . When the frequency of a pure tone stimulus was shifted from $750 \mathrm{~Hz}$ below the unit's best frequency to $750 \mathrm{~Hz}$ above, the discharge activity escalated from $10 \%$ to $100 \%$ of maximal response. The activity remained at this high level across a range of another $2-3 \mathrm{kHz}$ above the best frequency. Maximal discharge rates were up to $350 \mathrm{spikes} / \mathrm{sec}$.

VOC inhibition neurons responded best at specific stimulus intensities and most preferred stimulus intensities between 45 and $85 \mathrm{~dB}$ SPL, which correspond to natural echo intensities. 

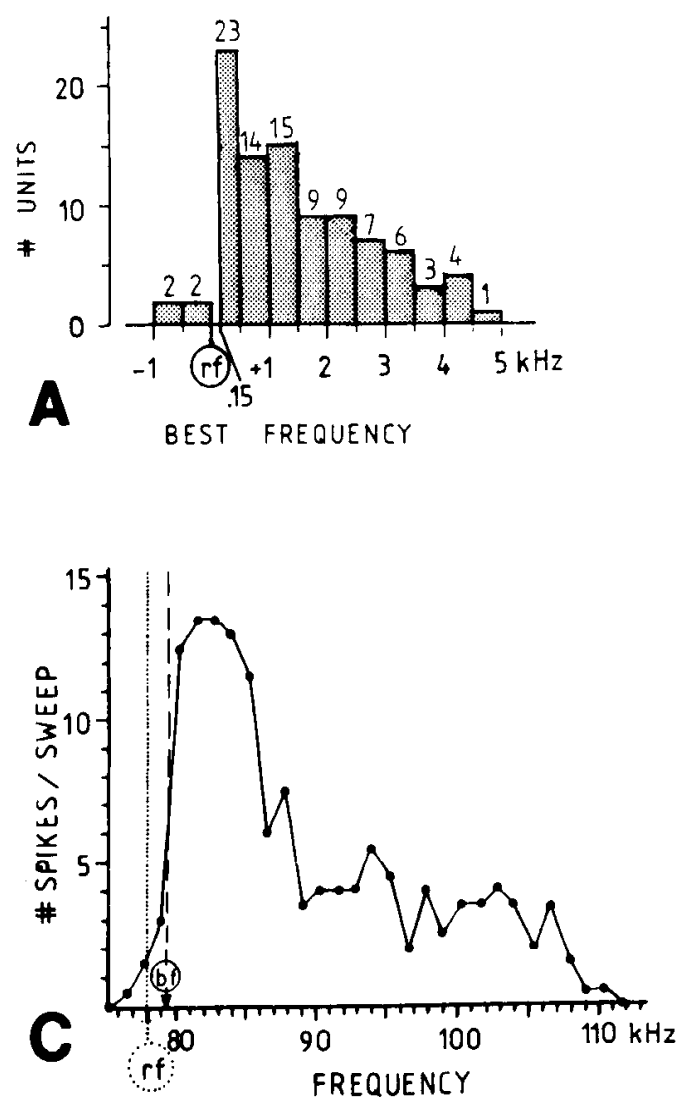
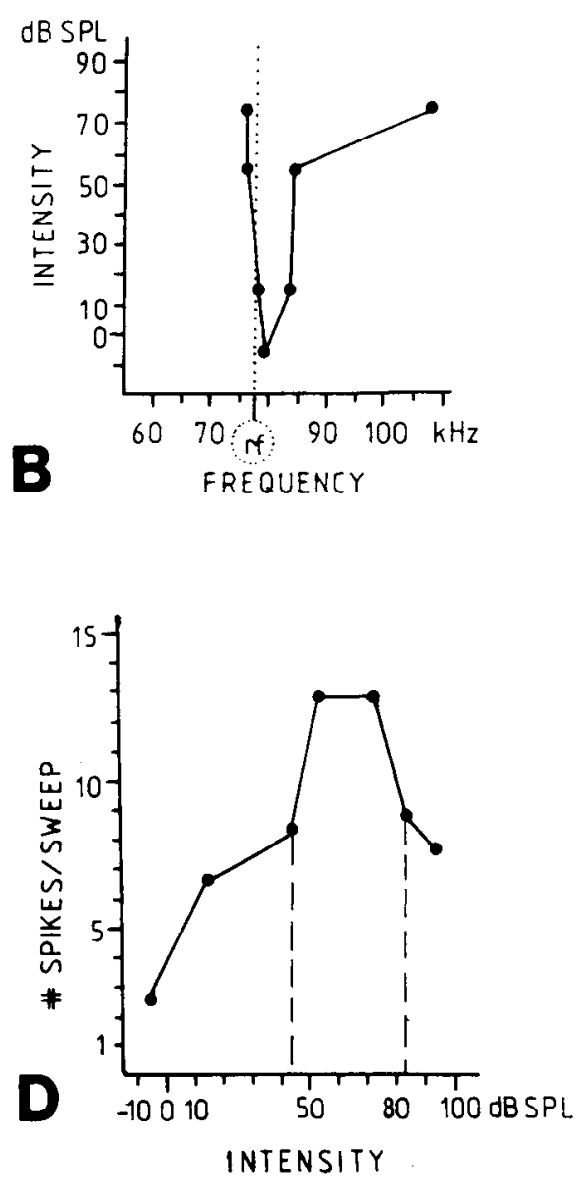

Figure 5. Response properties of VOC inhibition neurons to auditory stimuli. $A$, Distribution of best frequencies. The number of units (numbers above each column) is plotted against the best frequency (class width, $0.5 \mathrm{kHz}$ ) relative to the bats' individual resting frequen$\operatorname{cies}(r f)$. $B$, Tuning characteristics. The response threshold for minimum excitation (ordinate) is plotted against the stimulus frequencies [ahscissa; frequency values are indicated relative to a normalized resting frequency of 78 $\mathrm{kHz}$ (broken line)]. $C$, Frequency-response curve. The discharge activity (ordinate) is shown as a function of the stimulus frequency (abscissa). The best frequency of the unit $(b f)$ is marked by the dashed line. $r f$, resting frequency (normalized to $78 \mathrm{kHz}$ and marked by the dotted line). The stimulus intensity was at $72 \mathrm{~dB}$ SPL. $D$, Intensity spike count function. The amount of activity is plotted against the stimulus intensity. The response maximum between about 45 and $85 \mathrm{~dB}$ SPL is indicated by broken lines.
A typical dynamic response characteristic is shown in Figure $5 D$.

The remaining four classes of AV units exhibited an excitatory response to both vocalization and acoustic stimuli. They are presented below according to the percentage of their occurrence in the paralemniscal tegmentum.

(2) VOC duration units. These units fired tonically for a period that was inversely correlated with the duration the echolocation call. VOC duration units constituted $20 \%$ of the AV units recorded (38 out of 189). Excitation began as much as $50 \mathrm{msec}$ prior to the onset of vocalization and lasted throughout the emission of an echolocation call. The shorter the orientation call, the more spikes fired prior and during the call, and vice versa. This is demonstrated by the example shown in Figure $7 A$. The negative correlation between neuronal activity and the duration of vocalization is shown quantitatively for seven units in Figure $7 B$. On average, a shortening of the echolocation call by $10 \mathrm{msec}$ corresponded to an increase in discharge activity of $1.4 \mathrm{spikes} / \mathrm{sec}$. There was no correlation between frequency of vocalization and the spike rate.

Figure 6. Frequency-response curves of three VOC inhibition neurons (squares, neuron $\mathrm{a}$; circles, neuron $\mathrm{b}$; and triangles, neuron c) that were tuned to different best frequencies (marked by asterisks). The amount of activity (ordinate) is plotted against different stimulus frequencies (abscissa) relative to the resting frequency ( $r$, dotted vertical line). Only those frequencies over which DSC occurs are plotted. For an example of a complete frequency-response curve, see Figure $5 C$.

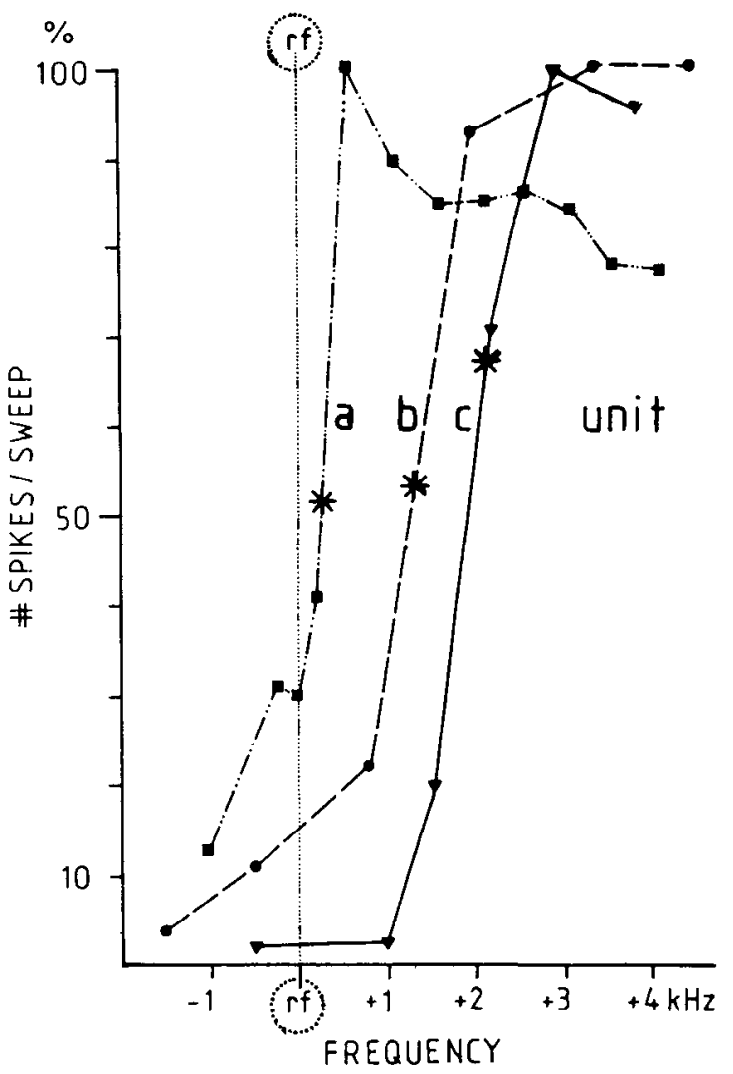



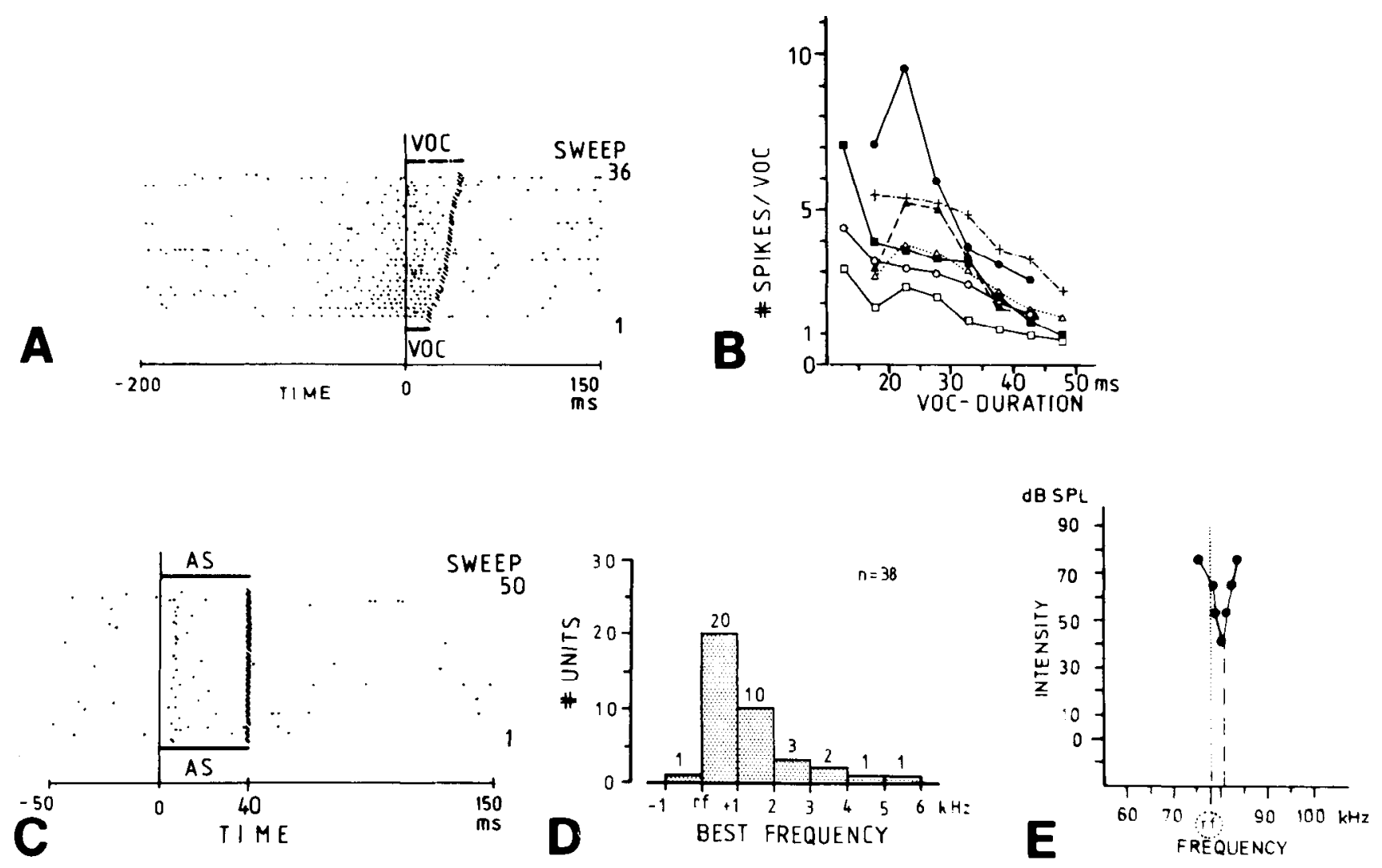

Figure 7. "VOC duration" neurons. Presentation of data in $A$ and $C-E$ is as in Figures 4 and 5 . $A$, These neurons raised their activity as early as $50 \mathrm{msec}$ before the vocalization onset and remained excited during the vocalization. The vocalizations are arranged according to increasing vocalization length from bottom to top. $B$, Relationship between the duration of emitted echolocation calls (abscissa) and the discharge activity (expressed by the number of spikes per vocalization, ordinate) for seven single units as indicated by different symbols. The "bin" width of vocalization durations was $5 \mathrm{msec}$. Vocalization durations shorter than $15 \mathrm{msec}$ and more than $50 \mathrm{msec}$ were summarized within one class each. $C$, Auditory stimuli identical to the vocalization elicited phasic-on discharges with latencies around $5 \mathrm{msec}$. $D$, Distribution of best frequencies relative to the bats' individual resting frequencies $(r f)$. The numbers above each column indicate the number of units within each class of best frequencies. $E$, Tuning characteristics. Minimum response thresholds (ordinate) are plotted against the stimulus frequencies (abscissa; rf, resting frequency normalized to $78 \mathrm{kHz}$ ). The best frequency is marked by the dashed line.

Acoustic signals that mimicked the bat's vocalization elicited a phasic-on response in VOC duration units (Fig. 7 C), at a mean latency of $5.1 \pm 1.6 \mathrm{msec}$. The majority of VOC duration units ( 30 out of 38) had best frequencies in the range between the bat's resting frequency and only $2 \mathrm{kHz}$ above (Fig. $7 D$ ). This frequency range matches closely the range of most Dopplershifted echo CF components. The tuning curves were symmetrical with minimum threshold values between 30 and $40 \mathrm{~dB}$ SPL (Fig. 7E).

(3) VOC frequency units. VOC frequency units discharged before and during vocalization, and their rate of discharge changed when the bat shifted its vocalization frequency. VOC frequency units made up $13 \%$ of the population of $A V$ neurons (25 out of 189). These units discharged tonically, beginning as early as $150 \mathrm{msec}$ prior to the beginning of an echolocation call, but during the emission of an orientation signal they were not active. The response pattern of a VOC frequency unit is shown in Figure $8 A$. When the bat lowered its vocalization frequency below the resting frequency, the discharge activity of VOC frequency neurons increased (Fig. $8 B$ ). A decrease of $1 \mathrm{kHz}$ in the frequency of the orientation call corresponded to an increase of 75 spikes/sec on average (ranging from 40 spikes/sec to 475 spikes/sec). No correlation between the duration of the vocalization and the spike rate was observed (Fig. $8 A$ ).
The response patterns of VOC frequency units to acoustic stimulation were heterogeneous. In 15 of the 25 VOC frequency neurons, acoustic signals elicited phasic-on excitation. One example is given in Figure $8 C$. The auditory responses of all VOC frequency units occurred at latencies that were much longer than in any other type of AV neurons. They averaged $10.5 \mathrm{msec} \pm$ $2.1 \mathrm{msec}$ (Fig. 8C). Of the 15 acoustically excited VOC frequency neurons, 13 were tuned to best frequencies between the resting frequency and $4 \mathrm{kHz}$ above it (Fig. $8 \mathrm{D}$ ). The remaining two neurons each had two best frequencies that were harmonically related to each other. Figure $8 E$ shows one of those doublepeaked tuning curves. The lower best frequencies of both of these neurons were situated just above the fundamental $\mathrm{CF}$ component of the bat's echolocation call around $39-40 \mathrm{kHz}$, and the upper best frequencies were above the bat's resting frequency around $79 \mathrm{kHz}$.

Acoustic stimulation of the remaining 10 of the 25 VOC frequency neurons suppressed their spontaneous activity. The suppression of spontaneous activity could be elicited by sounds with frequencies over a wide range, from 20 to $90 \mathrm{kHz}$ including the resting frequency. In fact, no best frequency for suppression could be determined for any of these units.

(4) VOC-off units. These units started firing only after the end of an emitted echolocation call. The tonic excitation began be- 

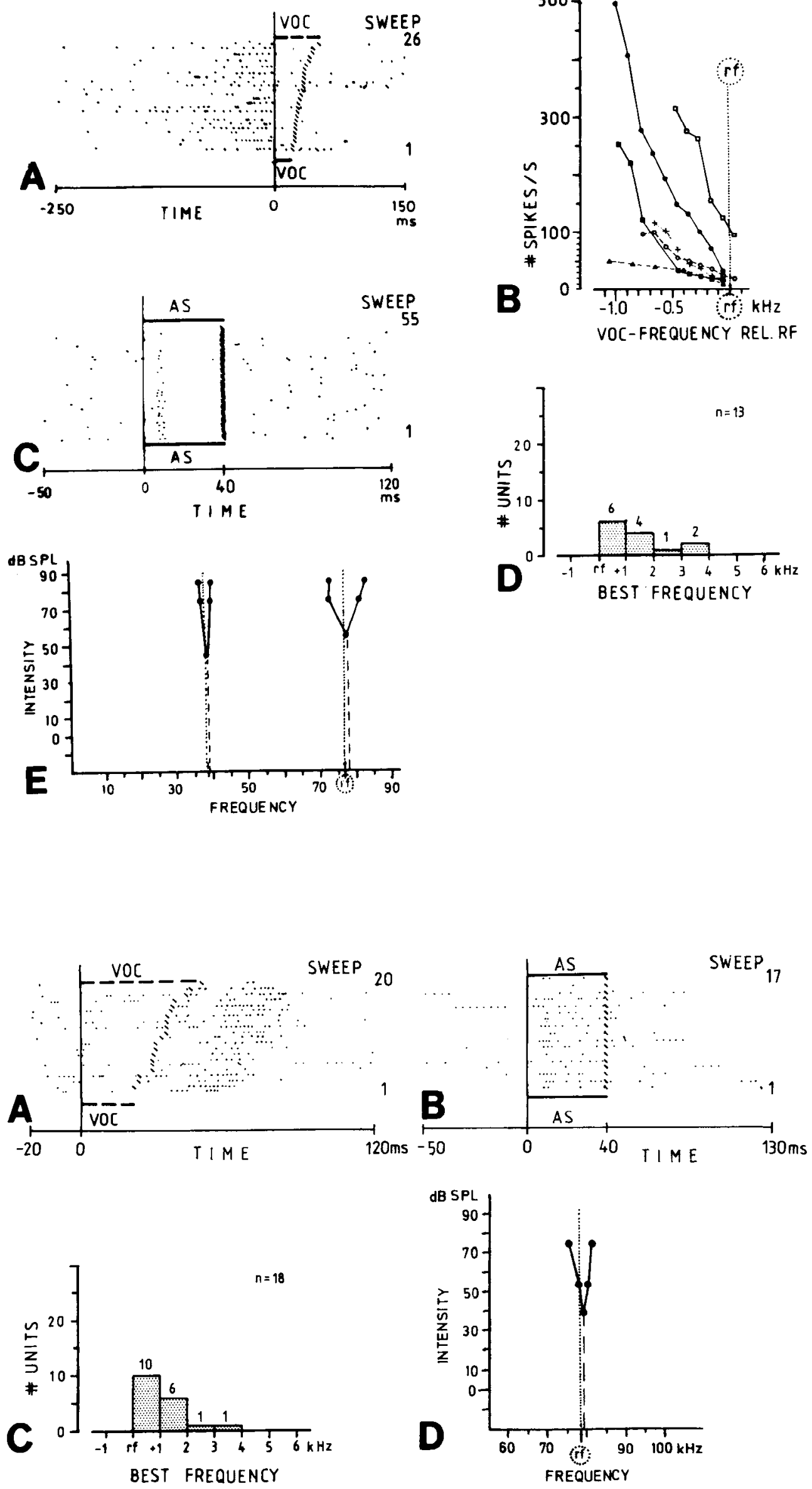

Figure 8. "VOC frequency" neurons. Presentation of data in $A$ and $C-E$ is as in Figures 4 and 5. $A$, The discharge rate increased up to $150 \mathrm{msec}$ before the beginning of an echolocation call and stopped with its onset. $B$, Discharge rates (ordinate, number of spikes per second) plotted against different frequencies of the CF component in echolocation calls (abscissa) relative to the bats' individual resting frequencies ( $r f$, vertical dotted line). Sample size is six units (indicated by different symbols). $C$, Auditory stimuli identical to the bat's vocalization elicited phasic-on responses, which habituated after the presentation of about 40 sweeps. $D$, Distribution of best frequencies plotted relative to the bats' resting frequencies $(r f) . E$, Tuning curve of a neuron with two minimum response thresholds (best frequencies indicated by dashed lines). The best frequencies were harmonically related to each other. $r f$, second harmonic frequency of the bat's echolocation call (= resting frequency, dotted line on the right); the fundamental frequency (dotted line on the left).
Figure 9. "VOC-off' neurons. Data presentation follows the convention of Figures 4 and 5. $A$, These neurons showed an excitatory activity starting between 15 and $25 \mathrm{msec}$ after the end of the vocalization. $B$, Auditory stimuli that were acoustically identical to the bat's vocalization elicited phasic tonic responses with latencies of about $7 \mathrm{msec}$. $C$, Distribution of best frequencies relative to the resting frequency $(r f) . D$, Tuning characteristics. The unit's minimum response threshold (ordinate) is plotted as a function of the stimulus frequency (abscissa). The resting frequency $(r f)$ is normalized to $78 \mathrm{kHz}$ and indicated by the dotted line. The best frequency of the unit is marked by the dashed line 
Figure 10. 'VOC-on-off' neurons. Displays of data are as in Figures 4 and 5. $A$, The neurons were excited around the beginning and around the end of the echolocation call. Before, during, and after the emission of an echolocation sound, the background activity ( $S P O)$ was reduced. $B$, The neurons responded to auditory response to stimuli identical to the bat's vocalization by a phasic discharge pattern. $C$, The distribution of best frequencies is given relative to the bats' individual resting frequencies $(r f)$. $D$, Tuning properties. The minimum response thresholds (ordinate) are given as a function of the stimulus frequencies (abscissa) relative to a normalized resting frequency ( $r$, dotted line) at 78 kHz. The best frequency of the unit is indicated by the dashed line.
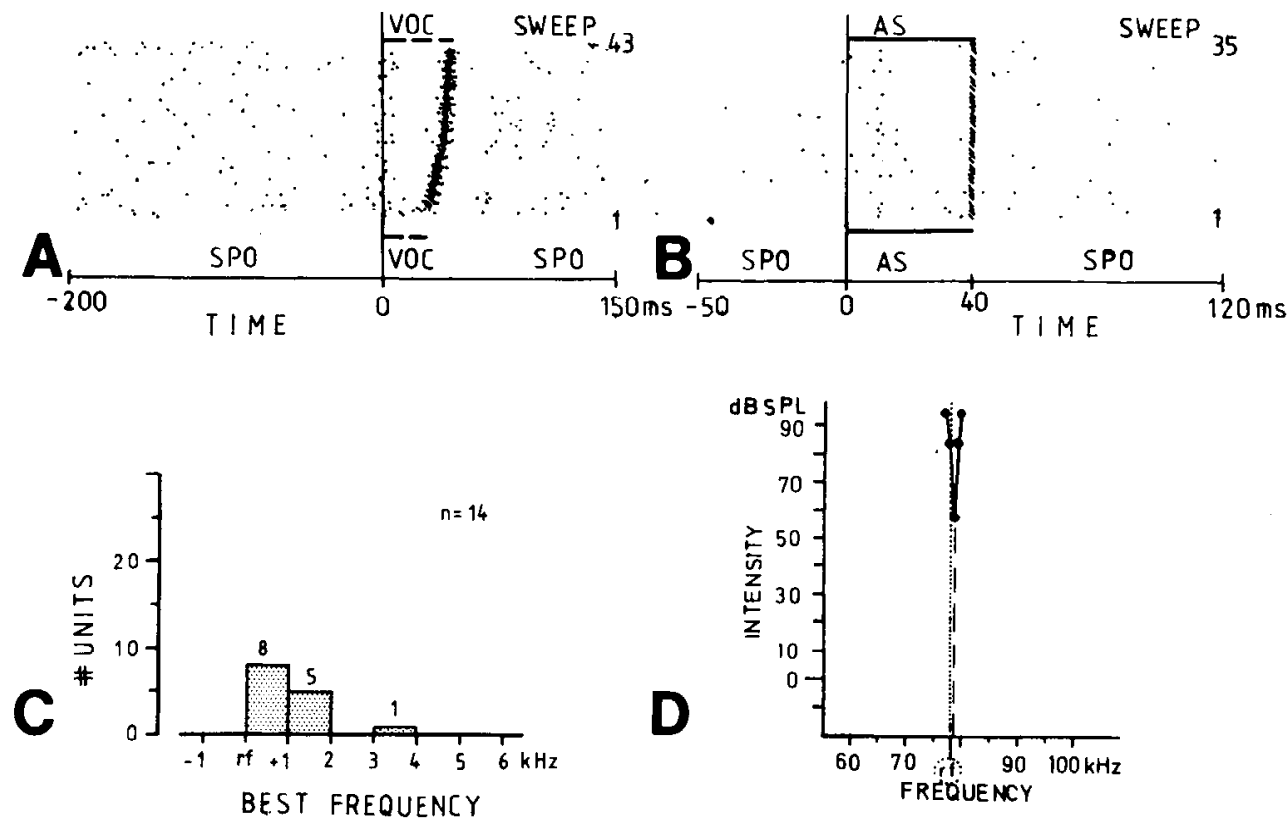

tween 15 and $25 \mathrm{msec}$ after the end of a vocalization and lasted for 20-30 msec (Fig. 9A); 10\% of all AV neurons displayed this type of activity (18 out of 189 ). The activity induced by the vocalization was not correlated with the beginning or with the duration or frequency of the echolocation call.

Acoustic signals that mimicked the orientation call elicited phasic-tonic excitation. This is demonstrated in Figure $9 B$. The latencies were $7.4 \mathrm{msec} \pm 1.3 \mathrm{msec}$ on average. The majority of VOC-off units (16 out of 18) had best frequencies concentrated in a frequency band between the bat's resting frequency and $2 \mathrm{kHz}$ above (Fig. 9C). This band covers the frequency range of echoes that elicit DSC. The tuning curves were symmetrical with minimum thresholds of 30-40 dB SPL (Fig. 9D).

(5) VOC-on-off units. VOC-on-off units responded with phasic excitation at the beginning and at the end of an echolocation call. The excitation occurred over a time span of $\pm 10 \mathrm{msec}$
VOC-inhibition

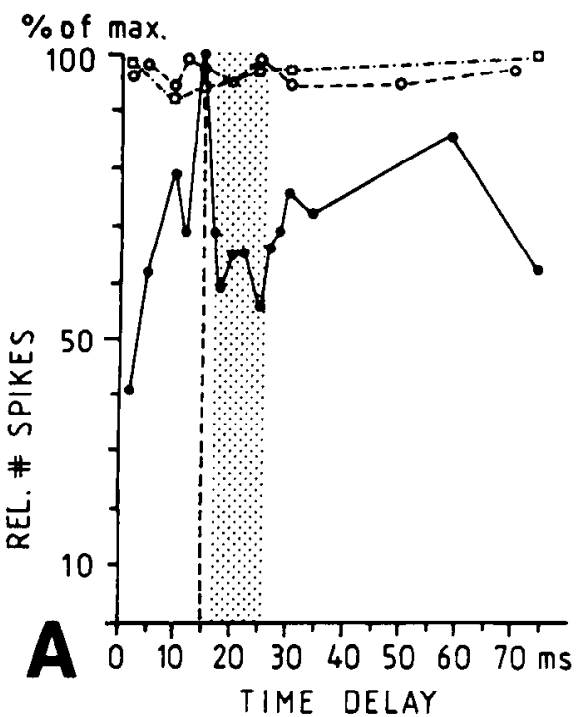

VOC-duration

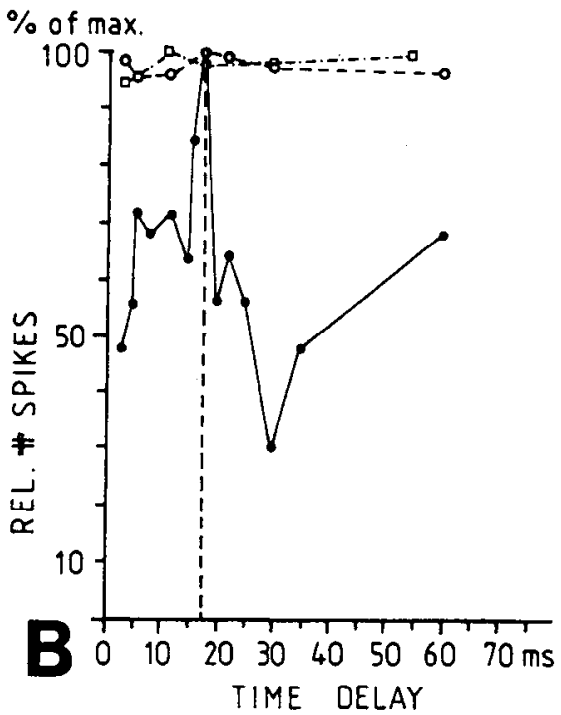

VOC-frequency

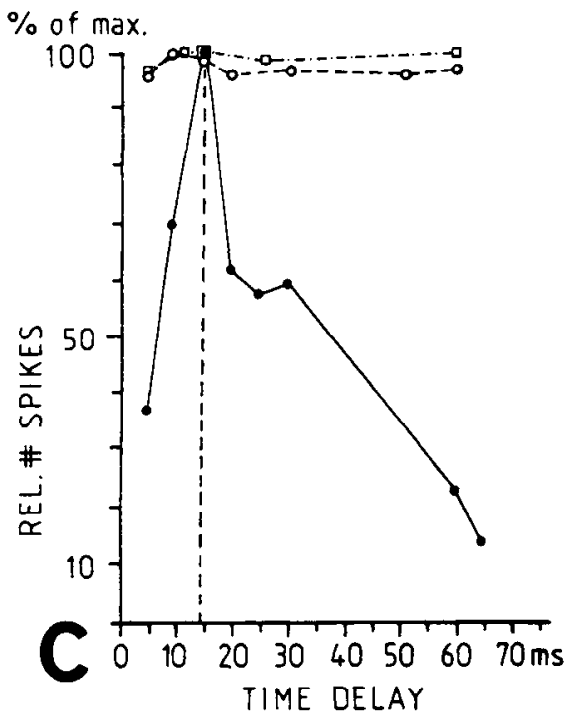

Figure 11. Responses to echo-simulating stimuli that were time locked to the beginning of a preceding vocalization sound depended upon the intervening time interval in VOC inhibition $(A)$, VOC duration $(B)$, and VOC frequency neurons $(C)$. This is indicated by the solid symbols in each of the three examples $(A-C)$. The discharge activity in each group is normalized with reference to maximal discharge (relative number of spikes, ordinate) and plotted against the time delay of the "echo" stimulus (abscissa). Distinct response maxima occurred in all three types of AV neurons (marked by the vertical broken lines). In all VOC inhibition neurons tested $(n=15)$, the auditory responses were reduced up to $50 \%$ between 18 and $25 \mathrm{msec}$ (shaded area in $A$ ). This distinct reduction at intervals greater than the optimal delay for excitation was not found in VOC duration or VOC frequency neurons. The delay sensitivity completely disappeared in all neurons when the act of vocalization was replaced by its auditory analog simulating the bat's vocalization. The "echo" responses are indicated by open squares when the vocalization-mimicking stimulus had its CF frequency at the fundamental frequency of the bat's echolocation signal, and by open circles when the CF frequency of the vocalization analog was at the second harmonic. The frequency of the "echo" stimulus was held at the units' best frequencies and at intensities between 30 and $50 \mathrm{~dB}$ above the minimum threshold. 


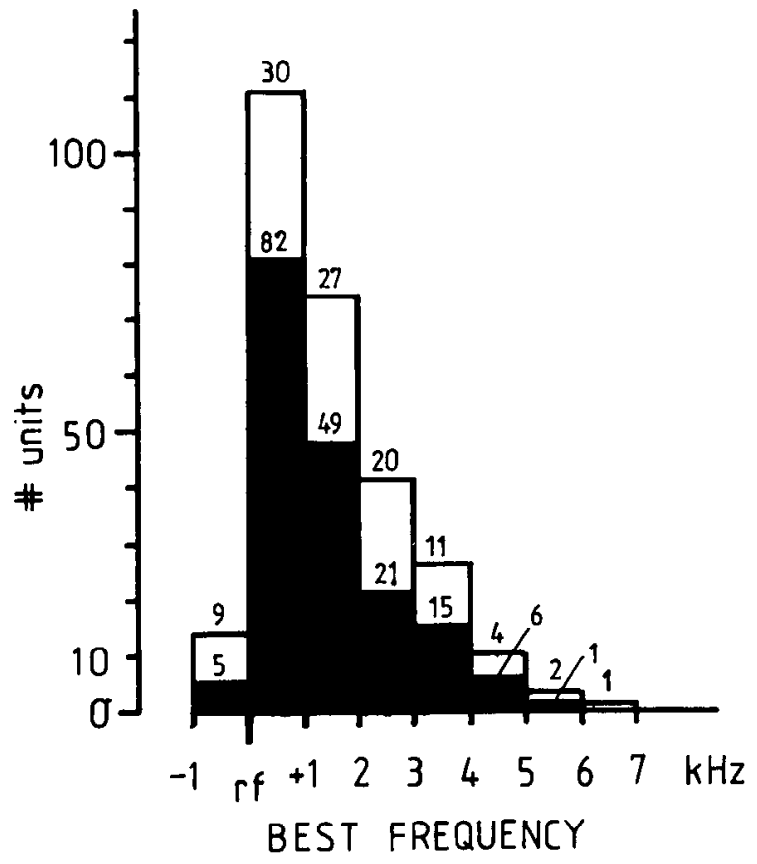

Figure 12. Distribution of best frequencies of paralemniscal neurons. Best frequencies could be determined in $179 \mathrm{AV}$ units (solid columns) and 104 auditory units (open columns). The numbers above the columns indicate the number of units within each class.

around the onset and the end of the vocalization, respectively. This activity pattern is illustrated by the unit shown in Figure $10 \mathrm{~A}$. Vocalization was correlated in time with suppression of spontaneous activity in VOC-on-off units. The suppression occurred during an interval of 20 and $80 \mathrm{msec}$ before the vocalization onset, between the excitatory responses to the onset and the end of an echolocation call, and between 20 and $40 \mathrm{msec}$ after the emission of an orientation signal. VOC-on-off neurons represented $7 \%$ of the total population of AV neurons (14 out of 189).

Acoustic stimuli that mimicked the bat's echolocation pulse elicited a phasic-on response with latencies of $8.5 \mathrm{msec} \pm 2.4$ $\mathrm{msec}$. The auditory response of a VOC-on-off neuron is shown in Figure 10B. Almost all VOC-on-off units (13 out of 14) had best frequencies ranging between the resting frequency and 2 $\mathrm{kHz}$ above it (Fig. 10C). The tuning curves werc symmetrical with minimum thresholds between 40 and $55 \mathrm{~dB}$ SPL (Fig. 10D).

The level of spontaneous activity of VOC-on-off neurons depended on whether the bat was vocally active or silent for more than about $1 \mathrm{~min}$. The spontaneous activity during sequences of echolocation calls was around 19 spikes/sec, but was only 3 spikes/sec when the bat did not vocalize (compare SPO in Fig. $10 A, B)$.

\section{Sensitivity of AV units to echo delay}

An echolocation situation was simulated by presenting a playback stimulus (echo mimic) locked in time to the emission of an echolocation call. When the time interval between the onset of an orientation call and the beginning of the echo mimic was varied, for some $A V$ units the magnitude of the response to the "echo" depended on the time delay. Out of a total of $41 \mathrm{AV}$ neurons tested, this delay sensitivity was observed in 25 neurons, which belonged to the VOC inhibition, the VOC duration,
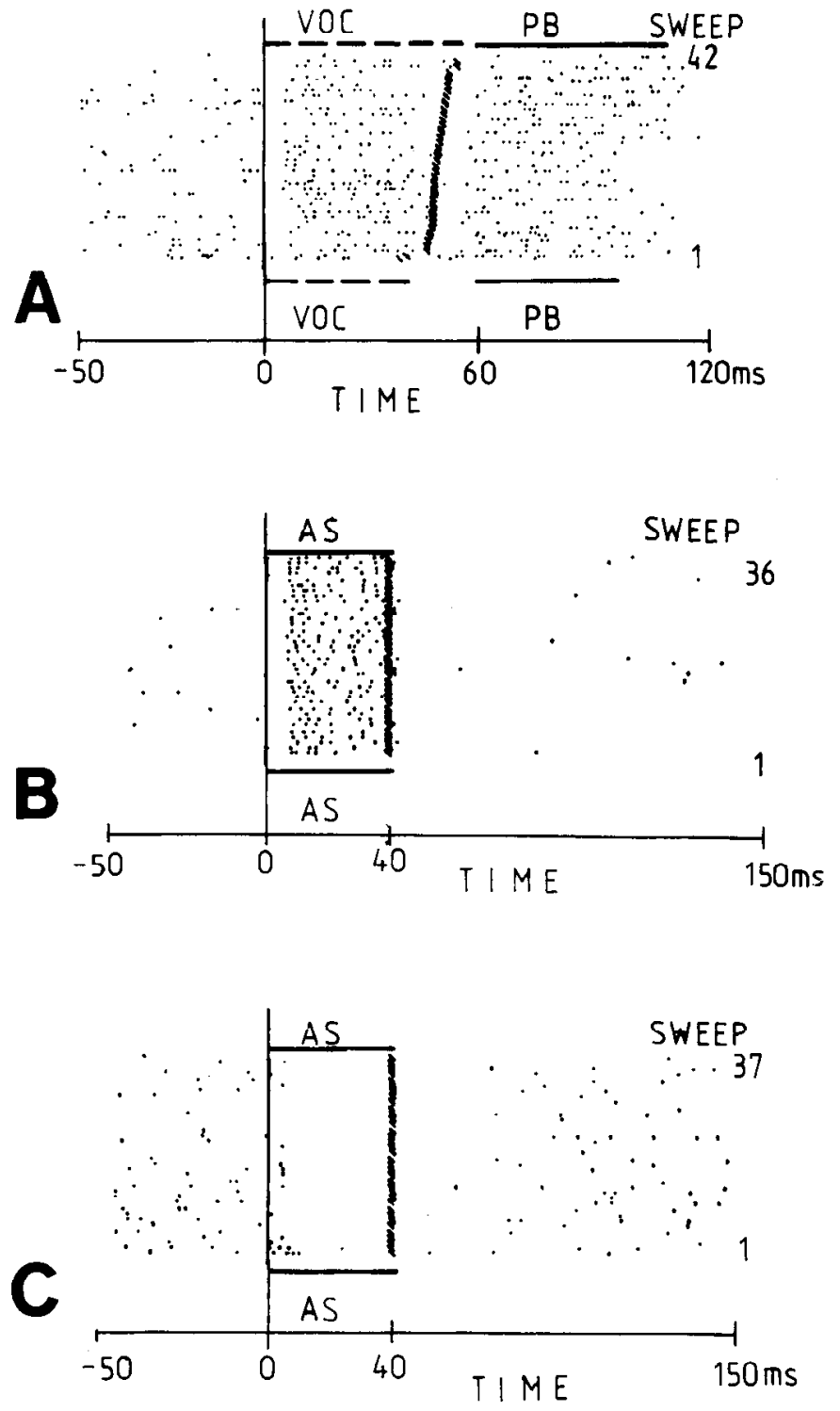

Figure 13. Paralemniscal auditory neuron with suppressive band. The displays follow the convention of Figure 4. $A$, The responses to the emission of an echolocation call ( VOC) and to an acoustically identical stimulus (playback signal, $P B$; delay, $60 \mathrm{msec}$ ) were the same. $B, A$ similar response was seen to an artificially generated auditory stimulus $(A S)$ when delivered at the unit's best frequency $(2.6 \mathrm{kHz}$ above the bat's resting frequency at an intensity of $73 \mathrm{~dB}$ SPL) and independently of the bat's sound emission. $C$, Auditory stimuli at a frequency only $0.9 \mathrm{kHz}$ above the resting frequency and below the unit's best frequency caused a suppression of background activity (stimulus intensity, $73 \mathrm{~dB}$ SPL).

and the VOC frequency type. For VOC inhibition and VOC frequency units, the delays at the response to the "echo" was maximum ( $=$ best delays) varied between 5 and $18 \mathrm{msec}$. In VOC duration units, the best delays varied between 15 and 30 msec. Figure 11 (solid symbols) illustrates the delay-sensitive echo responses of single units of the VOC inhibition (Fig. 11A), the VOC duration (Fig. $11 B$ ), and the VOC frequency type (Fig. $11 C$ ). Each of them showed a clear maximum response at a particular time delay ( $15 \mathrm{msec}$ in $A, 18 \mathrm{msec}$ in $B$, and $14 \mathrm{msec}$ in $C$ ). Additionally, in all of the VOC inhibition units tested, the echo response was reduced by as much as $50 \%$ at delays between 18 and $25 \mathrm{msec}$ (Fig. $11 \mathrm{~A}$, shaded area). 

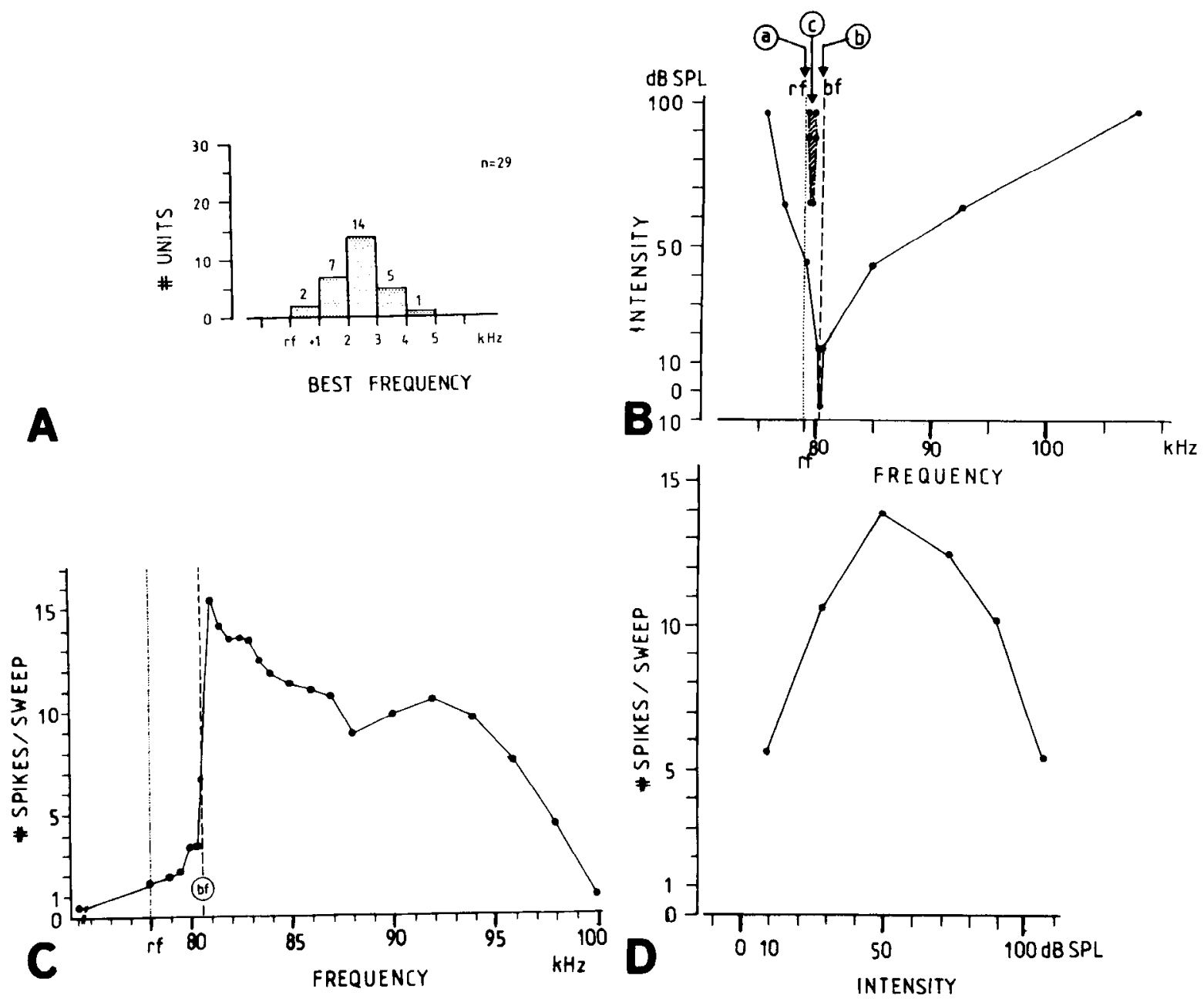

Figure 14. Response properties of the same paralemniscal auditory unit with suppressive band shown in Figure 13. Presentation of data is as in Figure 5. $A$, Distribution of best frequencies relative to the resting frequency $(r f)$. $B$, Tuning characteristics. The minimum thresholds for eliciting auditory responses (ordinate) are plotted against the stimulus frequency (abscissa). The best frequency (bf, dashed line) was $2.6 \mathrm{kHz}$ above the resting frequency ( $r f$, dotted line). Arrows $a-c$ indicate stimulus frequencies used in Figure $13 A-C$. Within the hatched area the background activity was suppressed (suppressive band). $C$, The discharge activity (ordinate) is shown as a function of the stimulus frequency ( $a b s c i s s a$ ). $b f$, best frequency (indicated by dashed line); $r f$, resting frequency (marked by the dotted line). $D$, The spike activity (ordinate) is plotted against the stimulus intensity (abscissa).

The sensitivity to particular echo delays completely disappeared when the vocalization that originally triggered the delayed presentation of the echo signal was replaced by an acoustic mimic of an orientation call. This is demonstrated in Figure 11 (open symbols) for the same AV units that exhibited delaysensitive responses when the echo was locked to the bat's vocalization (see Fig. 11, solid symbols). The frequency of the call's mimic was either at the fundamental frequency (Fig. 11, open squares) or at the second harmonic (Fig. 11, open circles). The frequency of the echo signal was maintained at the unit's best frequency. The magnitude of the echo response did not depend on the time interval between the acoustic simulation of the bat's vocalization and the echo signal.

Thus, the sensitivity of the echo response to particular time intervals between call and echo critically depends on the act of vocalization. The delay sensitivity cannot be elicited by the auditory reception of the vocalization.

\section{Auditory units}

Unlike AV units, auditory units did not show any correlation of their responses with active vocalization, and responded to emitted echolocation calls with discharge patterns and spike rates that were very similar to responses elicited by acoustic stimuli that mimicked the echolocation call; 118 units of this type were found within the paralemniscal tegmentum, and constituted $35 \%$ of all paralemniscal neurons recorded. However, depending on the stimulus frequency the discharge activity varied between different excitatory patterns. Even suppression of spontaneous activity occurred.

The discharge patterns of most paralemniscal auditory units ( $75 \%$ or 89 out of 118 ) resembled those of units of the nuclei of the lateral lemniscus (Metzner and Radtke-Schuller, 1987). However, most paralemniscal auditory units had best frequencies in the range of the Doppler-shifted CF components of the echo signal that the bat would hear before it compensated for them. The distribution of best frequencies within the entire population of paralemniscal auditory units is summarized in Figure 12 (open columns). The majority ( $91 \%$ or 95 units) had best frequencies that were clustered between the resting frequency and $7 \mathrm{kHz}$ above.

One-fourth of the paralemniscal auditory units $(25 \%$ or 29 out of 118) had response characteristics that were unlike those 
of units in the nuclei of the lateral lemniscus. The firing pattern of a paralemniscal auditory unit with an unusual response type is illustrated in Figure 13. Its discharge activity during vocalization was indistinguishable from the response to a playback vocalization, in both cases it was tonically excited (Fig. 13A, VOC and PB). However, the spontaneous background activity was suppressed by auditory stimuli at frequencies within a very narrow band between the unit's best frequency and the bat's resting frequency (Fig. 13C).

The majority of paralemniscal auditory units with suppressive bands ( 26 out of 29 ) had best frequencies that were concentrated in a range farther above the resting frequency (between $1 \mathrm{kHz}$ and $4 \mathrm{kHz}$ ) than the best frequencies of most other paralemniscal neurons (Fig. 14A; compare with Figs. $5 A, 7 D, 8 D, 9 C, 10 C$, 12). Only two of these units had best frequencies immediately above the resting frequency. Thus, this population of paralemniscal auditory neurons with suppressive bands (Figs. 13, 14) appears to be tuned to echoes that are frequency shifted by larger Doppler effects.

The responses of paralemniscal auditory units with suppressive bands (Fig. 14B-D) resembled those found in VOC inhibition units (compare with Fig. 5). Much as in VOC inhibition units, their tuning curves for the excitatory response had shallow slopes for the high-frequency side and sharp slopes on the lowfrequency side of the curve (Fig. 14B; compare with Fig. $5 B$ ). However, just below the unit's best frequency, but above the resting frequency of the bat, spontaneous activity was suppressed. This is indicated by the hatched area in Figure 14B. The discharge patterns for auditory stimuli that were presented at the bat's resting frequency, at the unit's best frequency, and within the area of suppression were already demonstrated by the examples given in Figure $13 A-C$, respectively.

These paralemniscal auditory neurons also responded with a striking change in their discharge rate when the frequency of an auditory stimulus was shifted around their best frequency. The frequency-response curve is plotted in Figure $14 C$. It is very similar to the one found in VOC inhibition units (compare with Fig. $5 C$ ). Frequency shifts of $\pm 750 \mathrm{~Hz}$ around the unit's best frequency caused a change in the firing rate between $10 \%$ and $100 \%$ of maximal response. The discharge activity remained at a high level even when the stimulus frequency rose far above the best frequency.

Finally, like VOC inhibition units, these auditory units responded best to stimulus intensities that match natural echo intensities. Figure $14 D$ shows a typical dynamic response curve (compare with Fig. $5 D$ for VOC inhibition units). The auditory units fired best between 30 and $80 \mathrm{~dB}$ SPL.

\section{Spatial distribution of paralemniscal neurons}

The AV neurons belonging to each of the five different AV types were not distributed uniformly over the whole paralemniscal area. Various types of AV units appeared to be clustered preferentially within different regions of the paralemniscal tegmentum. Figure 15 shows the distribution of the five classes of AV units.

The most obvious topography was secn for VOC frequency and VOC-off units, which predominated in the rostral portion of the paralemniscal tegmentum (Fig. 15A-C). Within this rostral paralemniscal region, VOC frequency neurons were located more dorsally and rostrally than VOC-off neurons.

VOC inhibition, VOC duration, and VOC-on-off units were found predominantly in the central and more caudal area of the

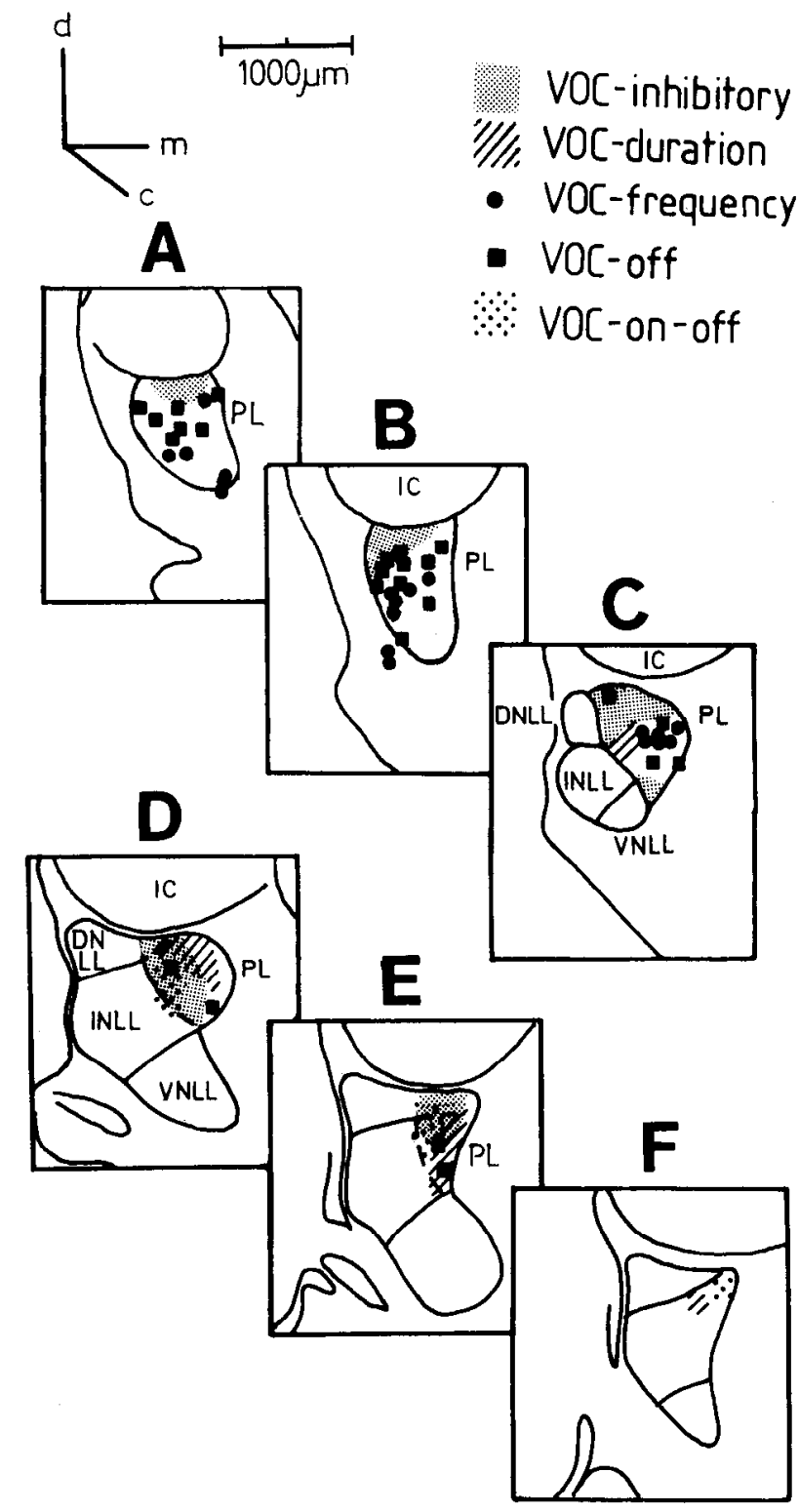

Figure 15. Schematic distribution of the AV neurons within the paralemniscal tegmentum in a frontal view. The sections $A-F$ are located at the same rostrocaudal levels as shown in Figure 2. DNLL, dorsal nucleus of the lateral lemniscus; $I C$, inferior colliculus; $I N L L$, intermediate nucleus of the lateral lemniscus; $P L$, paralemniscal tegmentum; $V N L L$, ventral nucleus of the lateral lemniscus.

paralemniscal tegmentum (Fig. 15D-F). VOC duration neurons seemed to be localized more medially than the VOC-on-off type, and VOC inhibition neurons occupied the dorsal portion of the rostral paralemniscal tegmentum. Within the extreme caudal paralemniscal tegmentum, however, they were found at greater depths as well. Auditory units were frequently found in the caudal portion of the paralemniscal tegmentum.

\section{Discussion}

\section{Vocalization and auditory processing interact at the} single-neuronal level

This study has demonstrated that individual neurons responded differently to a sound emitted by the bat than they did to identical acoustic signals played back to the bat. These neurons were called AV units. In a simulation of the bat's echolocation, the 
response of AV neurons to the simulated echo mimic appeared to depend on the time interval between the emission of an orientation call and the simulated echo. This delay sensitivity was triggered by the act of vocalization and not by auditory selfstimulation during call emission. This clearly indicates that there is interaction at the level of single ncurons between the pathways for motor control of vocalization and auditory processing. The AV interaction results in a gating of acoustic information by the preceding vocalization.

Only a few studies so far have reported AV neural activity in mammals, and even fewer have addressed this issue at the single-cell level. In two pioneering studies, Suga and Schlegel (1972) and Suga and Shimozawa (1974), demonstrated that vocalization influenced auditory processing in mammals. In the gray bat they found that evoked potentials at the level of the nuclei of the lateral lemniscus or the superior olivary complex were attenuated during the emission of an orientation signal compared to passive acoustical stimulation.

Most interesting is the report by Schuller (1979), who described two neurons in the inferior colliculus of the greater horseshoe bat that were excited only when vocalization and an acoustic stimulus occurrcd simultancously. In two other ncurons, he found that synchronization of neuronal activity to follow small frequency modulations similar to an echo returning from a fluttering insect occurred only when the bat was vocalizing. In another 26 neurons, vocalization qualitatively altered the responses to acoustic stimuli. Suga and Yajima (1989) also described qualitative changes of discharge patterns during vocalization in the mustache bat. They found such neurons within the lateral portions of the periaqueductal gray and the adjacent reticular formation.

In the squirrel monkey, Müller-Preuss and Ploog (1981) and Müller-Preuss (1989) found single neurons that reduced their spontaneous activity during the monkey's phonation. The same neurons were excited by acoustic stimulation. These neurons were found adjacent to the central nucleus of the inferior colliculus, near the medial geniculate body, and in the auditory cortex.

In most of these studies the vocalization signals were elicited by electrical stimulation of areas very close to the recording sites. Thus, it is not possible to exclude that the stimulation might have influenced the neuronal activity at the recording sites. To avoid any artifacts from electrical stimulation, in the study presented here the bats emitted vocalizations spontaneously.

\section{Paralemniscal neurons may control vocalization during DSC}

The discharge patterns and firing rates of paralemniscal neurons are influenced by almost all parameters of echolocation calls and echo signals that occur during DSC.

Virtually all (95\%) of the paralemniscal neurons sampled were most sensitive in the frequency range of Doppler-shifted echoes. The finding that no VOC inhibition units had best frequencies between the resting frequency and $150 \mathrm{~Hz}$ above is consistent with the fact that echo frequencies between the resting frequency and $50-300 \mathrm{~Hz}$ above it fail to initiate DSC (Schnitzler, 1968; Schuller et al., 1974).

The firing rates of VOC inhibition units as well as of paralemniscal auditory units with suppressive bands were affected by even very small frequency shifts around their best frequency. This is consistent with the high degree of accuracy of DSC. Schuller et al. (1974) have demonstrated that horseshoe bats compensate for Doppler-shifted echoes with an accuracy of approximately $50-300 \mathrm{~Hz}$. This is only about $0.1 \%$ of the bat's resting frequency.

VOC inhibition and paralemniscal auditory units with suppressive bands responded best to stimulus intensities that correspond closely to intensities of natural echoes (see Simmons, 1973).

VOC inhibition and VOC frequency units responded best to a simulated echo when it was presented with a time delay not greater than $18 \mathrm{msec}$ after the emission of an orientation call. The response of VOC inhibition units to the echo mimic was markedly reduced when it was delivered later than $18 \mathrm{msec}$. Behavioral experiments (Schuller, 1974, 1977; Neumann and Schuller, 1991) have demonstrated that only echoes that return with delays less than $20 \mathrm{msec}$ after the onset of a vocalization initiate DSC in horseshoe bats. Echoes that return later fail to induce DSC.

VOC duration and VOC frequency neurons are selective for either the duration or the pure tone frequency of the bat's echolocation calls. Both duration and frequency are systematically changed during DSC (Schnitzler, 1968; Schuller, 1977), although they may vary independently. The vocalization frequency may be lowered without varying call length (e.g., Schnitzler, 1968), and changes in sound duration may occur in behavioral contexts other than DSC (e.g., Neuweiler et al., 1987).

In VOC frequency neurons, lower vocalization frequencies were correlated with higher firing rates. In the motor nucleus of laryngeal control, the nucleus ambiguus, and in the superior laryngeal nerve, however, lower vocalization frequencies are encoded by lower spike rates (Schuller and Rübsamen, 1981; Rübsamen and Betz, 1986). Therefore, the frequency code of the paralemniscal VOC frequency neurons would have to be inverted if these neurons were to transfer their frequency information to motor commands in the nucleus ambiguus. This hypothesis is supported by the fact that the vast majority of paralemniscal neurons stain for glutamic acid decarboxylase (M. Vater, personal communication). That suggests that they may bc GABAcrgic, and through inhibition accomplish the postulated inversion of the spike rate code.

Direct evidence for the control of vocalization by paralemniscal neurons derives from several electrical stimulation studies. In a recent investigation in the rufous horseshoe bat, Schuller and Radtke-Schuller (1990) could elicit echolocation signals by microelectrical stimulation within the paralemniscal tegmentum. Suga et al. (1973) elicited species specific echolocation calls in an area anteroventral to the inferior colliculus in a variety of different families of bats (Vespertilionidae, Mormoopoidae, and Noctilionidae). These stimulation sites were located in an area that corresponds to the paralemniscal nuclei in horseshoe bats.

\section{A model for the control of vocalization frequency due to inhibitory auditory feedback}

VOC inhibition neurons in the paralemniscal tegmentum and paralemniscal auditory neurons with suppressivc bands are the only neurons known so far that could provide all of the sensory information necessary for horseshoe bats to lower their vocalization frequency through auditory feedback. Figure 16 illustrates how these neurons could accomplish that.

In this model, the echo signal excites the VOC inhibition neurons (neurons $X$ and $Y$ in Fig. 16) and the paralemniscal auditory neurons with suppressive bands (neuron $Z$ in Fig. 16). Let us assume that the VOC inhibition neuron $\mathrm{X}$ had the lowest 


\section{motor side}

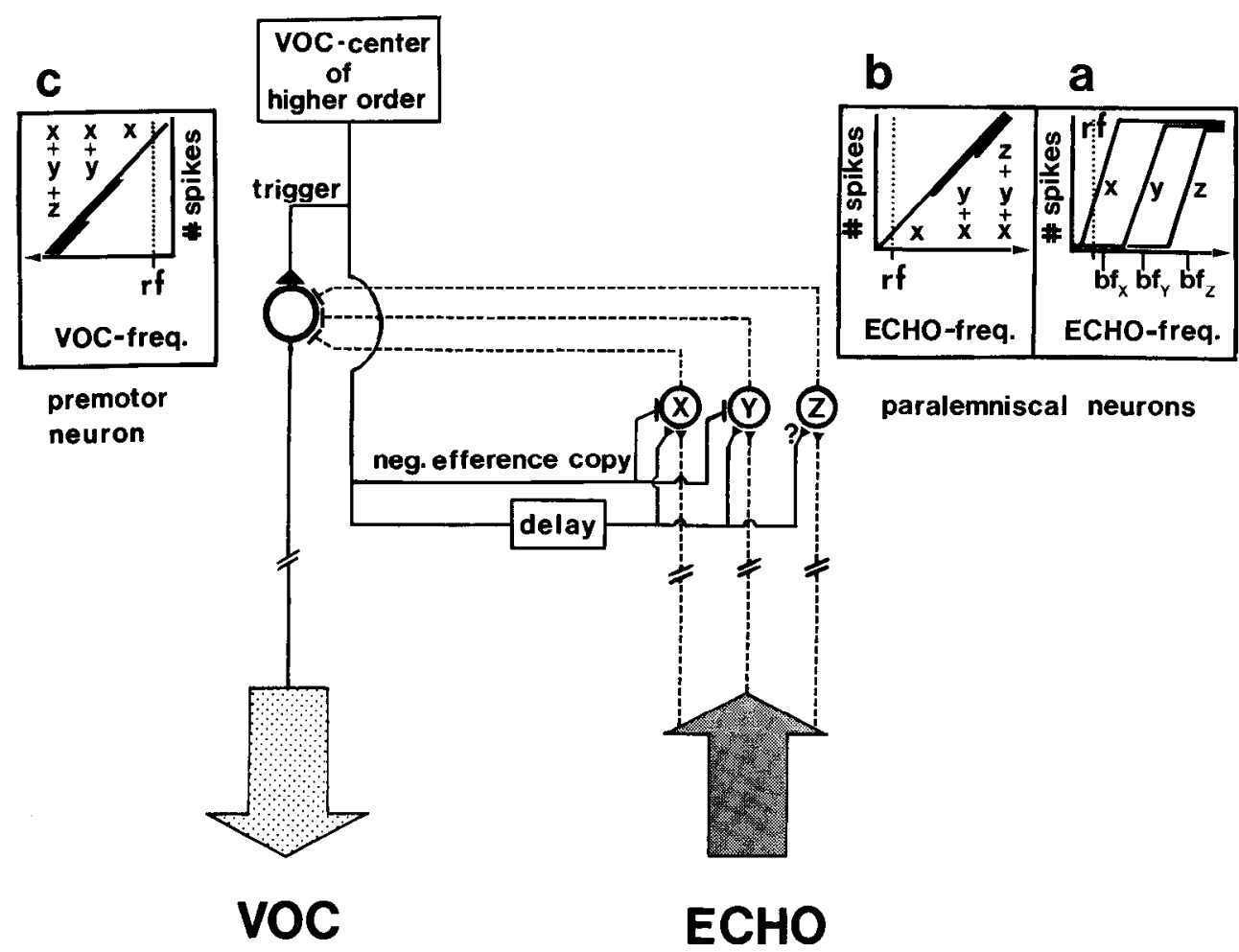

sensory side

Figure 16. Model for the regulation of the vocalization frequency (motor output, left) due to auditory information provided by VOC inhibition and paralemniscal auditory neurons with suppressive bands (sensory input, right). For details, see Discussion. best frequency just above the resting frequency $\left(\mathrm{bf}_{\mathrm{x}}\right.$ in inset $a$ ), and the paralemniscal auditory neuron $Z$ had the highest bcst frequency $\left(\mathrm{bf}_{\mathrm{z}}\right.$ in inset $a$ ). From the frequency-response curves of these neurons, we know that increasing echo frequencies above the resting frequency elicit rises in the activity until a maximum rate is attained (inset $a$; see also Figs. $5 C, 6,14 C$ ). The firing rate then remains at a high level even for a continuing frequency increase. With echo frequencies increasing just above the resting frequency, the neuron responding first is the neuron with the lowest best frequency (neuron $X$ in Fig. 16). The farther the echo frequency rises above the resting frequency, the more neurons with successively higher best frequencies will be recruited. After neuron $X$ follows the VOC inhibition neuron $Y$, and finally, the auditory neuron $Z$ joins the population of neurons that are excited by frequency-shifted echo signals.

The total activity of the whole population of paralemniscal neurons $\mathrm{X}, \mathrm{Y}$, and $\mathrm{Z}$ is given in inset $b$ of Figure 16. First, the activity increases due to an increase in firing of neuron $X$; then, the rising discharge activity of neuron $Y$ is added; finally, neuron $\mathrm{Z}$ contributes with its increasing discharges to the population's total activity. The amount of total activity could then be used to determine the deviation of the echo signal from the resting frequency.

To generate the appropriate motor command for the compensation of Doppler-shifted echoes, it is assumed that the population of paralemniscal neurons $\mathrm{X}, \mathrm{Y}$, and $\mathrm{Z}$ converge via inhibitory synapses onto premotor or motoneurons (left side in Fig. 16). These motor or premotor neurons have a high rate of discharge when echoes are at the resting frequency, that is, when the activity in the paralemniscal population is low. If projections from the paralemniscal neurons $\mathrm{X}, \mathrm{Y}$, and $\mathrm{Z}$ are inhibitory, higher spike rates in the paralemniscal neuronal population would lower the spike rates in premotor or motoneurons (inset $c$ in
Fig. 16) and thus lower the vocalization frequency. This is the idea of an inhibitory auditory feedback mechanism.

The information about the deviation of the echo frequency from the resting frequency would have to be stored to affect the subsequent vocalizations (Schuller and Suga, 1976). The site of storage of the echo information is not yet known.

However, VOC inhibition neurons and paralemniscal auditory neurons with suppressive bands can only account for the initial decrease in vocalization frequencies in response to echo frequencies shifted above the bat's reference frequency. Once the bat compensated, the echoes return at the reference frequency that no longer excites VOC inhibition neurons sufficiently (see the "gap" in their distribution of best frequencies shown in Fig. $5 A$; see also Fig. 6). Therefore, they are unable to provide the negative feedback necessary to maintain the vocalization frequency below the resting frequency as long as the bat is in flight. The vocalization frequency should consequently return to the resting frequency if only VOC inhibition neurons and paralemniscal auditory neurons with suppressive bands were involved. On the other hand, echo frequencies around the reference frequency are particularly well encoded by a large number of "classical" auditory neurons. For instance, approximately one-third of all neurons recorded within the dorsal nucleus of the lateral lemniscus had best frequencies in the narrow range between the resting frequency and $1 \mathrm{kHz}$ above it (Metzner and Radtke-Schuller, 1987). These neurons could provide the negative feedback necessary to inhibit the activity of premotor neurons as long as the echo frequency returns at the reference frequency, that is, the compensating bat continues to fly at a particular speed.

Any increase in flight velocity creates a frequency shift in the subsequent echo signal above the reference frequency that would yield a de novo excitation of VOC inhibition and of paralem- 
niscal auditory units with suppressive bands. Their activity could provide the negative feedback that is added to the stored information about the previous frequency deviation. The system could therefore be compared with a "sample and hold" circuit as already suggested by Schuller and Suga (1976).

If the bat reduccs its flight spced, the echo frequency decreases below the reference frequency and the bat adjusts by increasing its vocalization frequency back toward the resting frequency. Behavioral experiments, however, suggest that the return to the resting frequency is not controlled by auditory feedback (Schuller, 1974, 1986). The DSC behavior in horseshoe bats appears to be composed of two separate systems. One system uses auditory feedback and controls the decrease of the vocalization frequency in response to increasing echo frequencies. The model proposed above would provide such a mechanism. On the other hand, the system that drives the vocalization frequency back toward the resting frequency appears to be independent of auditory feedback and operates continuously even when the echo frequency is at or below the bat's resting frequency.

Figure 16 also illustrates a model to explain the discharge activity of VOC inhibition neurons. During the emission of an ccholocation call, the background activity of VOC inhibition neurons is suppressed. No auditory stimulus, even when acoustically identical to the bat's vocalization, elicited an inhibitory response in VOC inhibition neurons. This indicates that suppression of background activity must be due to a negative efference copy (von Holst and Mittelstaedt, 1950) of the bat's echolocation call. A negative neuronal "image" of the vocalization would suppress the background activity of VOC inhibition neurons (neurons $X$ and $Y$ in Fig. 16), whenever an echolocation call is emitted. This might serve to improve the neuronal signal-to-noise ratio. Most of the VOC inhibition units had best frequencies very close to the bat's resting frequency and therefore would be excited by acoustic self-stimulation during the emission of an echolocation call. This could mask the response to the subsequent echo.

The delay-dependent responses of VOC inhibition neurons (ncurons $\mathrm{X}$ and $\mathrm{Y}$ in Fig. 16) to simulated echoes following vocalizations could be generated by a delayed excitatory neuronal copy of the bat's vocalization that gates the auditory response for echoes returning at distinct time delays. The echo stimulus together with the delayed excitatory neuronal copy of the bat's echolocation call could overcome the suppression of neuronal activity that is caused by the negative efference copy of the vocalization. However, such a pathway for delayed neuronal images of the bat's vocalization remains to be demonstrated.

The idea that vocalization frequencies are controlled by some kind of inhibitory feedback mechanism is supported by deafening experiments in cats and observations in deaf humans. The fundamental frequency of vocalizations in cats increases after the loss of auditory feedback (Shipley et al., 1988). Speaking deaf humans also tend to speak in a voice that contains higher fundamental frequencies than in hearing human speakers (Boothroyd et al., 1975; Forner and Hixon, 1977; Leder et al., 1987; Waldstein, 1992; W. Metzner, unpublished observation). They are even reported to speak in falsetto voice. However, when adequate auditory feedback was provided, as with a cochlear implant, the fundamental frequency was one of the earliest acoustic features that approached normal values again (Leder et al., 1986). Psychoacoustic experiments in humans also demonstrated that the fundamental frequency of the voice increased when an artificially delayed auditory feedback was presented (Fairbanks, 1955).

Studies in the squirrel monkey show that, in primates, much as in horseshoe bats (Schuller and Rübsamen, 1981), the frequency of emitted vocalizations is negatively correlated with the activity of motoneurons (Larson and Kistler, 1986). Increased vocalization frequencies in deaf cats and humans, therefore, can only be explained by a loss of inhibition from the sensory side. In terms of the proposed model (Fig. 16), the removal of the auditory input (right side) would eliminate any inhibition of the premotor "resting" activity (left side) in two ways. First, the loss of auditory input eliminates any auditory activity of paralemniscal neurons $X, Y$, and $Z$ even at the resting frequency. Second, the suppression of background activity in the VOC inhibition neurons $X$ and $Y$ during the act of vocalization is still active. Therefore, the vocalization frequency would no longer be held at the resting frequency but would instead increase above the bat's resting frequency.

Some influence of cortical structures on the performance of DSC is suggested by recent experiments in the mustached bat, another bat species compensating for Doppler-shifted echoes (Riquimaroux et al., 1991). Pharmacological lesions within an area of the auditory cortex that contains neurons tuned to Doppler-shifted echo frequencies altered the bat's frequency discrimination performance. After application of muscimol, a GABA agonist, the bats were no longer able to discriminate pulse-echo pairs with frequency differences between 50 and $500 \mathrm{~Hz}$. The discrimination performance for frequency differences greater than $500 \mathrm{~Hz}$, however, was not disturbed. In contrast, in horseshoe bats, lesions of vast cortical areas including the auditory cortex had no influence on DSC behavior (Movchan, 1984). In the same series of experiments it was shown that DSC disappeared after lesioning deep layers of the inferior colliculus and ventrally adjacent structures. These lesions included large portions of the paralemniscal tegmentum.

In another study using mustached bats, Gooler and O'Neill (1988) found a topographic representation of vocalization frequencies within a cortical area that most probably corresponds to the anterior cingulate cortex (Jürgens and Pratt, 1979). However, lesions of this cortical region did not change DSC behavior (Gaioni et al., 1988).

Echolocating horseshoe bats are not the only mammals that adjust their vocalization frequency in response to frequency shifts in the auditory feedback. Psychoacoustic experiments demonstrated that even humans compensated for frequency shifts in playbacks of their vocalizations (Elman, 1981): when the frequency of the auditory feedback was artificially shifted upward or downward by up to $15 \%$ of the fundamental frequency, the test subjects responded by lowering and increasing their fundamental vocalization frequencies, respectively. Most individuals were even unaware of the fact that the feedback had been altered; awareness of the frequency-shifted feedback, however, did not prevent the compensation behavior. This suggests that the control of vocalization frequencies in response to auditory feedback may be accomplished by an evolutionary ancient neural mechanism common among all mammals.

\section{References}

Boothroyd A, Archambault P, Adams RE, Storm RD (1975) Use of a computer based-system of speech analysis and display in a remedial speech program for deaf child. Volta Rev 77:178-193.

Bruns V (1976) Peripheral auditory tuning for fine frequency analysis 
by the CF-FM bat, Rhinolophus ferrumequinum. II. Frequency mapping in the cochlea. J Comp Physiol 106:87-97.

Casseday JH, Covey E, Vater M (1988) Connections of the superior olivary complex in the rufous horseshoe bat, Rhinolophus rouxi. $\mathrm{J}$ Comp Neurol 278:313-329.

Covey E, Hall WC, Kobler JB (1987) Subcortical connections of the superior colliculus in the mustache bat, Pteronotus parnellit. J Comp Neurol 263:179-197.

Elman JL (1981) Effects of frequency-shifted feedback on the pitch of vocal productions. J Acoust Soc Am 70:45-50.

Fairbanks $G$ (1955) Selective vocal effects of delayed auditory feedback. J Speech Hearing Dis 20:333-346.

Forner LL, Hixon TJ (1977) Respiratory kinematics in profoundly hearing-impaired speakers. J Speech Hearing Res 20:373-407.

Gaioni SJ, Suga N, Riquimaroux H (1988) Effects of bilateral ablation of the auditory cortex and/or cingulate cortex on the biosonar behavior of the mustache bat. Soc Neurosci Abstr 14:442.7.

Gooler DM, O'Neill WE (1987) Topographic representation of vocal frequency demonstrated by microstimulation of anterior cingulate cortex in the echolocating bat, Pteronotus parnellii. J Comp Physiol 161:283-294.

Henkel CK, Edwards SB (1978) The superior colliculus control of pinna movements in the cat: possible anatomical connections. J Comp Neurol 182:763-776.

Jürgens U, Pratt R (1979) The cingular vocalization pathway in the squirrel monkey. Exp Brain Res 34:499-510.

Larson CR, Kistler MK (1986) The relationship of periaqueductal gray neurons to vocalization and laryngeal EMG in the behaving monkey. Exp Brain Res 63:596-606.

Leder SB, Spitzer JB, Milner P, Flevaris-Phillips C, Richardson F, Kirchner JC (1986) Re-acquisition of contrastive stress in an adventitiously deaf speaker using a single-channel cochlear implant. J Acoust Soc Am 79:1967-1974.

Leder SB, Spitzer JB, Kirchner JC (1987) Speaking fundamental frequency of postlingually profoundly deaf adult men. Ann Otol Rhinol Laryngol 96:322-324.

Metzner W (1988a) Neuronal interaction of vocalization and auditory processing in horseshoe bats. In: Sense organs-interfaces between environment and behavior (Elsner N, Barth FG, eds), p 27. Stuttgart: Thieme.

Metzner W (1988b) A neuronal audio-vocal interface in horseshoe bats. 11 th Annual Meeting European Neuroscience Association 27:7.

Metzner W (1989) A possible neuronal basis for Doppler-shift compensation in echolocating horseshoe bats. Nature 341:529-532.

Metzner W, Radtke-Schuller S (1987) The nuclei of the lateral lemniscus in the rufous horseshoe bat, Rhinolophus rouxi. J Comp Physiol [A] 160:395-411.

Movchan EV (1984) Participation of auditory centers in echolocative tracking of a moving target by greater horseshoe bat. Neurobiologia 16:737-745 (in Russian).

Müller-Preuss P (1989) Neural correlates of audio-vocal behavior: properties of anterior limbic cortex and related areas. In: Physiological control of mammalian vocalization (Newman JD, ed), pp 245-261. New York: Plenum.

Müller-Preuss P, Ploog D (1981) Inhibition of auditory cortical neurons during phonation. Brain Res 215:61-76.

Neumann I, Schuller G (1991) Spectral and temporal gating mechanisms enhance the clutter rejection in the echolocating bat, Rhinolophus rouxi. J Comp Physiol [A] 169:109-116.

Neuweiler G (1970) Neurophysiologische Untersuchungen zum Echoortungssystem der Großen Hufeisennase Rhinolophus ferrumequinum. Z Vgl Physiol 67:273-306.

Neuweiler G, Vater M (1977) Response patterns to pure tones of cochlear nucleus units in the CF-FM bat Rhinolophus ferrumequinum. J Comp Physiol 115:119-133.

Neuweiler G, Metzner W, Heilmann U, Rübsamen R, Eckrich M, Costa HH (1987) Foraging behavior and echolocation in the rufous horseshoe bat (Rhinolophus rouxi) of Sri Lanka. Behav Ecol Sociobiol 20: 53-67.

Obrist M (1989) Individuelle Variabilitüt der Echoortung: Vergleichende Freilandunter-suchungen an vier vespertilioniden Fledermausarten Kanadas. PhD thesis, University of Munich.

Ostwald J (1984) Tonotopic organization and pure tone response characteristics of single units in the auditory cortex of the greater horseshoe bat. J Comp Physiol [A] 155:821-834.
Paxinos G, Watson C (1986) The rat brain in stereotaxic coordinates 2d ed. Sydney: Academic.

Pietsch G, Schuller G (1987) Auditory self-stimulation by vocalization in the CF-FM bat, Rhinolophus rouxi. J Comp Physiol [A] 160:635644.

Riquimaroux H, Gaioni SJ, Suga N (1991) Cortical computational maps control auditory perception. Science 251:565-568.

Rübsamen R, Betz M (1986) Control of echolocation pulses by neurons of the nucleus ambiguus in the rufous horseshoe bat, Rhinolophus rouxi. I. Single unit recordings in the ventral motor nucleus of the laryngeal nerves in spontaneously vocalizing bats. J Comp Physiol [A] 159:675-687.

Schnitzler HU (1968) Die Ultraschallortungslaute der Hufeisen-Fledermäuse (Chiroptera, Rhinolophidae) in verschiedenen Orientierungssituationen. Z Vgl Physiol 57:376-408.

Schuller G (1974) The role of overlap of echo with outgoing echolocation sound in the bat Rhinolophus ferrumequinum. Naturwissenschaften 61:171-172.

Schuller G (1977) Echo delay and overlap with emitted orientation sounds and Doppler-shift compensation in the bat Rhinolophus ferrumequinum. J Comp Physiol 114:103-114.

Schuller G (1979) Vocalization influences auditory processing in collicular neurons of the CF-FM bat, Rhinolophus ferrumequinum. J Comp Physiol 132:39-46.

Schuller G (1986) Influence of echolocation pulse rate on Doppler shift compensation control system in the greater horseshoe bat. $J$ Comp Physiol [A] 158:239-246.

Schuller G, Pollak GD (1979) Disproportionate frequency representation in the inferior colliculus of Doppler compensating greater horseshoe bats: evidence of an acoustic fovea. J Comp Physiol 132:47-54.

Schuller G, Radtke-Schuller S (1990) Neural control of vocalization in bats: mapping of brainstem areas with electrical microstimulation eliciting species-specific echolocation calls in the rufous horseshoe bat. Exp Brain Res 79:192-206.

Schuller G, Rübsamen R (1981) Laryngcal ncrve activity during pulse emission in the CF-FM bat, Rhinolophus ferrumequinum. I. Superior laryngeal nerve (external motor branch). J Comp Physiol 143:317321 .

Schuller G, Suga N (1976) Storage of Doppler-shift information in the echolocation system of the 'CF-FM' bat, Rhinolophus ferrumequinum. J Comp Physiol 105:9-14.

Schuller G, Beuter K, Schnitzler HU (1974) Response to frequency shifted artificial echoes in the bat Rhinolophus ferrumequinum. J Comp Physiol 89:275-286.

Schuller G, Beuter K, Rübsamen R (1975) Dynamic properties of the compensation system for Doppler shifts in the bat Rhinolophus ferrumequinum. J Comp Physiol 97:113-125.

Schuller G, Radtke-Schuller S, Betz M (1986) A stereotaxic method for small animals using expcrimentally determined reference profiles. J Neurosci Methods 18:339-350.

Shipley C, Buchwald JS, Carterette EC (1988) The role of auditory feedback in the vocalization of cats. Exp Brain Res 69:431-438.

Simmons JA (1973) The resolution of target range by echolocating bats. J Acoust Soc Am 54:157-173.

Suga N, Schlegel P (1972) Neural attenuation of responses to emitted sounds in echolocating bats. Science 177:82-84.

Suga N, Shimozawa T (1974) Site of neural attenuation of responses to self-vocalized sounds in echolocating bats. Science 183:1211-1213.

Suga N, Yajima Y (1989) Auditory-vocal integration in the midbrain of the mustached bat: periaqueductal grey and reticular formation. In: The physiological control of mammalian vocalization (Newman JD, ed), pp 87-108. New York: Plenum.

Suga N, Schlcgel P, Shimozawa T, Simmons J (1973) Orientation sounds evoked from echolocating bats by electrical stimulation of the brain. J Acoust Soc Am 54:793-797.

Trappe M, Schnitzler HU (1982) Doppler-shift compensation in insect-catching horseshoe bats. Naturwissenschaften 69:193-194.

Vater M, Feng AS, Betz M (1985) An HRP-study of the place-frequency map of the horseshoe bat cochlea: morphological correlates of the sharp tuning to a narrow frequency band. J Comp Physiol [A] 157:671-686.

von Holst E, Mittelstaedt H (1950) Das Reafferenzprinzip. Naturwissenschaften $37: 464-476$.

Waldstein RS (1992) Effects of postlingual deafness on speech production: implications for the role of auditory feedback. J Acoust Soc Am 88:2099-2114. 